M. Abdelwahed, N. Chorfi*, and R. Malek

\title{
Reconstruction of Tesla micro-valve using topological sensitivity analysis
}

https://doi.org/10.1515/anona-2020-0014

Received November 18, 2018; accepted January 14, 2019.

Abstract: In this paper, we deal with topology optimization attributed to the non stationary Navier-Stokes equations. We propose an approach where we analyze the sensitivity of a shape function relating to a perturbation of the flow domain. A numerical optimization algorithm based on topological gradient method is built and applied to the 2D Tesla micro valve reconstruction. Some numerical results confirm the efficiency of the proposed approach.

Keywords: Non-stationary Navier-Stokes equations, Tesla valve, Shape optimization, Topological sensitivity method

\section{Introduction}

Tesla valves are no-moving-part valves that utilize fluidic inertial forces to inhibit flow in the reverse direction. It was patented in 1920 by Nikola Tesla as a "Valvular conduit" [1] (see figure 1), and has since made the subject of various applications in micro-satellite [2], drug delivery [3], microbiology [4, 5] and hydrocephalus treatment in medicine $[6,7]$.

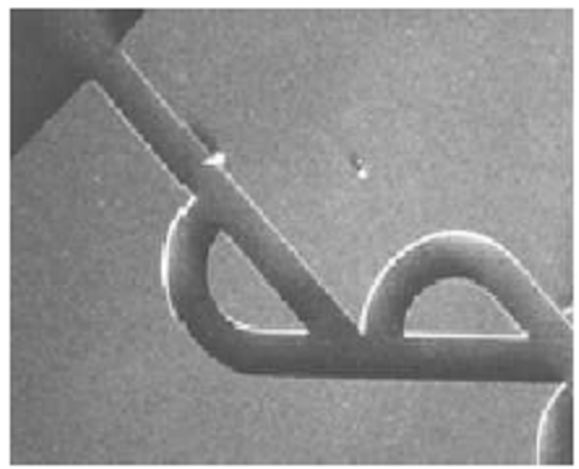

Fig. 1: A rotated scanning electron microscope photograph of a Tesla valve by Forster et al. [8]

The Tesla micro-valve performance is evaluated by the diodicity parameter (represents the ratio of the pressure drop in backward and forward direction) which evaluates the ability of allowing forward flow while

M. Abdelwahed, Department of Mathematics, College of Sciences, King Saud University, Saudi Arabia

*Corresponding Author: N. Chorfi, Department of Mathematics, College of Sciences, King Saud University, Saudi Arabia, E-mail: nchorfi@ksu.edu.sa

R. Malek, FSM, Department of Mathematics, Monastir University, Tunisia 
inhibiting the reverse one,

$$
D i=\frac{\Delta p_{\text {backward }}}{\Delta p_{\text {forward }}} .
$$

Different works have focused on the optimal shape of the tesla micro-valve. However, the majority of works concerns stationary Partial Differential Equations (PDE). Forster et al. [8] proved the possibility of using Tesla valves in micro-fluidics and determined experimentally the diodicity for Reynolds number $(\mathrm{Re}) \simeq$ 180. Truong et al. in [9] derived numerically the optimum geometry of Tesla valve for $100<R e<600$ with better diodicity than [8]. Bardell et al [10] analyzed the mechanism of the diodicity and proposed a Tesla valve optimal design for low Re. In the case when $R e=100$, they finished with $D i=1.4$. Gamboa et al. [11] optimized the shape of Tesla valve for application with piezoactuated plenums. The obtained fluid domain related to $R e=100$ is characterized by a diodicity number $D i=1.1$. In 2008, Pingen et al. [12] used the Lattice Bolzmann Method for the optimization of a micro Tesla valve without any information on diodicity. The used objective function was the pressure drop between inlet and outlet. After that in 2010, Lin et al. [13] used a topology optimization technique based on the power dissipation energy [14] of forward flow as objective function and the diodicity was built into the model as a constraint. For $R e=100$, they found a new design of Tesla valve given $D i=1.2$. Next in 2015, Lin et al. [15] solved the Tesla valve topology optimization using the approach of material distribution with inverse diodicity as objective function and fluid volume fraction as the constraint.

Until recently, there were no investigations dealing with the non-stationary case. We propose in this paper a new reconstruction method using the sensitivity analysis approach [16-19] for a non stationary flow.

The principal results of this work concern both theoretical and numerical aspects associated with the Tesla micro-valve problem. The theoretical part is related to the analysis of the topological sensitivity for the non stationary Navier-Stokes equations. The numerical part concerns the 2D optimization of the Tesla microvalve shape. The optimal shape is constructed by inserting obstacles in the considered initial domain. We build a simple and fast numerical reconstruction algorithm based on the topological gradient technique. The efficiency of the presented approach is confirmed by some numerical tests.

The paper is presented as following: Firstly we formulate the problem in section 2. Section 3 concerns the theoretical aspects. The numerical aspects are given in section 4. Finally section 5 includes Theorems proofs.

\section{Problem formulation}

Let $\Omega \subset \mathbb{R}^{d}, d=2,3$ a bounded domain with regular boundary $\Gamma=\partial \Omega$. We consider the blood as an incompressible viscous fluid flow described by the non stationary Navier-Stokes equations [20]. The velocity $w$ and the pressure $p$ satisfy the following system:

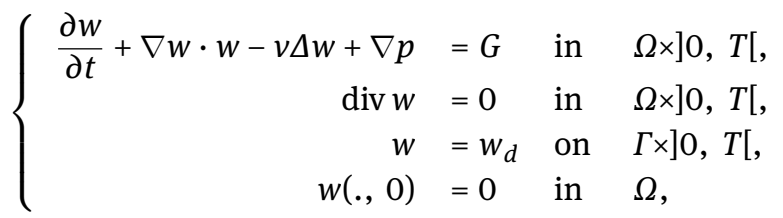

where $v$ is the kinematic viscosity coefficient, $G$ is the gravitational force, $T$ is the computational time and $w_{d}$ is a given Dirichlet boundary data. Because of the divergence free condition on $w, w_{d}$ must necessarily satisfy the compatibility condition,

$$
\left.\int_{\Gamma} w_{d}(x, t) . n d s(x)=0, \quad \text { a.e. } t \in\right] 0, T[
$$

where $n$ is the unit outward normal vector along $\Gamma$. 
Remark 2.1. Problem (1) has at least one solution (see [21](Ch.II,eq.(1.89)). If $|w|_{1, \Omega}<v / k$, with

$$
k= \begin{cases}\frac{2 \sqrt{2}}{3} \operatorname{meas}(\Omega)^{1 / 6} & \text { if } \quad d=3 \\ \frac{1}{2} \operatorname{meas}(\Omega)^{1 / 2} & \text { if } \quad d=2\end{cases}
$$

then problem (1) has a unique solution (see [17]).

The topological sensitivity method idea is to study the variation of a given shape function $j$ relating to a perturbation in the fluid flow domain geometry.

In structural shape optimization case (respectively electromagnetism and fluid dynamics cases) a geometry perturbation means removing some material (respectively the insertion of an obstacle).

Let $\mathcal{O}_{z, \varepsilon}=z+\varepsilon \mathcal{O}$, a small obstacle inserted in $\Omega$ characterized by its center $z$, its size $\varepsilon$ and its shape $\mathcal{O}$. $\mathcal{O}$ is a bounded domain of $\mathbb{R}^{d}$ containing the origin and $\partial \mathcal{O}$ (its boundary) is connected and piecewise $\mathcal{C}^{1}$.

The shape function variation is written

$$
j\left(\Omega \backslash \overline{\mathcal{O}_{z, \varepsilon}}\right)-j(\Omega)=\rho(\varepsilon) \delta j(z)+o(\rho(\varepsilon)), \forall z \in \Omega,
$$

where

- $\quad \varepsilon \mapsto \rho(\varepsilon)$, a positive scalar function going to zero with $\varepsilon$

- $\quad z \mapsto \delta j(z)$, called the topological gradient, describes the shape function variation when an obstacle is inserted in $z$. It plays the role of descent direction in the algorithm of optimization.

To our knowledge, the majority of works leading with topological sensitivity method concern the stationary case such as Stokes problem [16, 18], quasi-Stokes [19], stationary Navier Stokes problem [17]. We extend this method to the nonlinear unsteady Navier Stokes flow. To overcome the difficulty due to the non linear operator and its associated adjoint problem we extend the perturbed velocity by zero in the inclusion which permits to use the adjoint method in the whole domain. For the time dependent term we will use the fundamental solution of the non stationary Stokes operator and decompose the velocity variation.

We define the time dependent shape function as:

$$
j\left(\Omega \backslash \overline{\mathcal{O}_{z, \varepsilon}}\right)=\int_{0}^{T} J_{\varepsilon}\left(w_{\varepsilon}(., t)\right) d t
$$

where $J_{\varepsilon}$ in $H^{1}\left(\Omega \backslash \overline{\mathcal{O}_{z, \varepsilon}}\right)^{d}$ and $w_{\varepsilon}$ is solution to

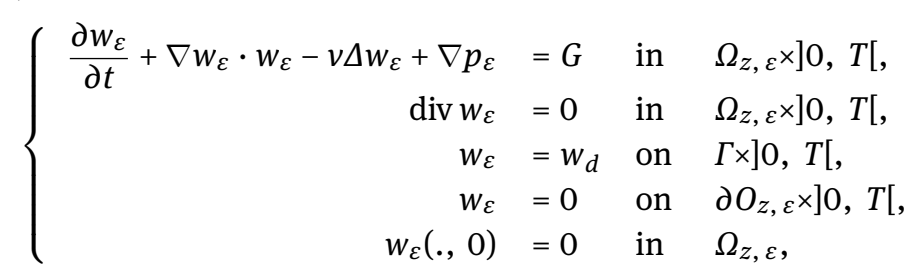

with $\Omega_{z, \varepsilon}=\Omega \backslash \overline{\mathcal{O}_{z, \varepsilon}}$ is the perturbed domain. Note that if $\varepsilon=0$ (without obstacle), ( $\left.w_{0}, p_{0}\right)$ verify (1) and $\Omega_{0}=\Omega$.

In the following, we will derive a general mathematical analysis for $J_{\varepsilon}$ satisfying the following assumption:

\section{Assumption $(\mathcal{A})$}

i) $\forall \varepsilon \geq 0, t \mapsto J_{\varepsilon}\left(w_{\varepsilon}(., t)\right) \in L^{1}(0, T)$.

ii) $J_{0}$ is differentiable in $H^{1}(\Omega)$ and we denote $D J_{0}(w)$ its derivative.

iii) $\exists \rho: \mathbb{R}_{+} \longrightarrow \mathbb{R}_{+}$and $\delta \mathcal{J} \in \mathbb{R}$ such that $\forall \varepsilon \geq 0$

$$
\int_{0}^{T}\left[J_{\varepsilon}\left(w_{\varepsilon}(., t)\right)-J_{0}\left(w_{0}(., t)\right)\right] d t=\int_{0}^{T} D J_{0}\left(w_{0}(., t)\right)\left(w_{\varepsilon}(., t)-w_{0}(., t)\right) d t+\rho(\varepsilon) \delta \mathcal{J}+o(\rho(\varepsilon)) .
$$




\section{Main results}

We deal in this section with the non stationary Navier-Stokes topological sensitivity relating to the domain perturbation. We consider the shape functions verifying the assumption $(\mathcal{A})$.

\subsection{Asymptotic behavior of the velocity variation}

We first study the influence on the velocity $v_{\varepsilon}=w_{\varepsilon}-w_{0}$ of inserting a small obstacle $O_{z, \varepsilon}$ in $\Omega$. From (1) and (3), it is straightforward to show that $\left(v_{\varepsilon}, p_{v_{\varepsilon}}\right)$ satisfy the system

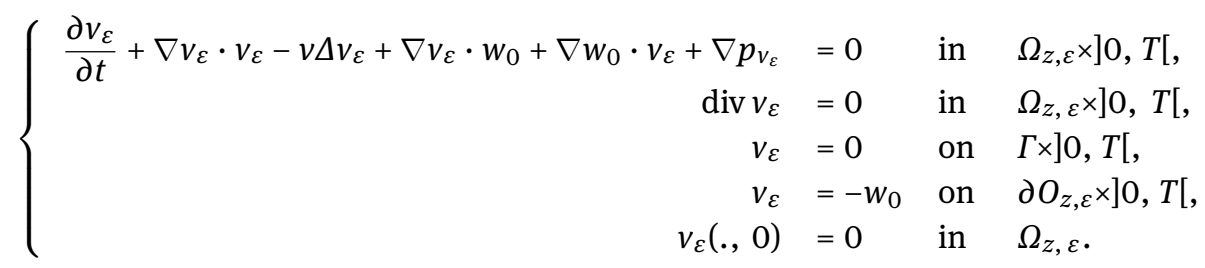

We will distinguish in the following the $2 D$ and $3 D$ cases.

\subsubsection{Three dimensional case}

Theorem 3.1. There exists $c>0$ independent of $\varepsilon$, such that

$$
\left\|v_{\varepsilon}(x, t)-W(x, t)\right\|_{L^{2}\left(0, T ; H^{1}\left(\Omega_{z, \varepsilon}\right)\right.} \leq c \varepsilon,
$$

where $W=\left(W^{1}, W^{2}, W^{3}\right) \in H^{1}\left(\Omega_{z, \varepsilon}\right)^{3}$ is defined by

$$
\left.W^{j}(x, t)=U^{j}\left(\frac{x-z}{\varepsilon}\right) \cdot w_{0}(z, t), \forall(x, t) \in \mathbb{R}^{3} \backslash \overline{\mathcal{O}}_{\varepsilon} \times\right] 0, T[,
$$

with $U^{j}$ is solution of (exterior Stokes problem)

$$
\left\{\begin{array}{rlll}
-v \Delta U^{j}+\nabla P^{j} & =0 & \text { in } & \mathbb{R}^{3} \backslash \overline{\mathcal{O}} \\
\operatorname{div} U^{j} & =0 & \text { in } & \mathbb{R}^{3} \backslash \overline{\mathcal{O}} \\
U^{j} & \longrightarrow 0 & \text { at } & \infty \\
U^{j} & =-e_{j} & \text { on } & \partial \mathcal{O},
\end{array}\right.
$$

with $\left\{e_{j}\right\}_{j=1,2,3}$ is the $\mathbb{R}^{3}$ canonical basis.

We show by using a single layer potential (see [22]) that

$$
U^{j}(y)=\int_{\partial \mathcal{O}} E(y-x) \eta_{j}(x) d s(x), \quad P^{j}(y)=\int_{\partial \mathcal{O}} \Pi(y-x) \eta_{j}(x) d s(x), \quad \forall y \in \mathbb{R}^{3} \backslash \overline{\mathcal{O}} .
$$

where

$$
E(y)=\frac{1}{8 \pi v r}\left(I+e_{r} e_{r}^{T}\right), \quad \Pi(y)=\frac{y}{4 \pi r^{3}} \forall y \in \mathbb{R}^{3} .
$$

with $r=\|y\|, e_{r}=\frac{y}{r}, e_{r}^{T}$ is the transpose of $e_{r}$ and $\eta_{j} \in H^{-1 / 2}(\partial \mathcal{O})^{3}$ is a solution of the boundary integral equation

$$
\int_{\partial \mathcal{O}} E(y-x) \eta_{j}(x) d s(x)=-e_{j}, \quad \forall y \in \partial \mathcal{O} .
$$

Using Theorem 3.1 we obtain the following corollary.

Corollary 3.2. We have

$$
\left.v_{\varepsilon}(x, t)=W(x, t)+O(\varepsilon), \quad x \in \Omega_{z, \varepsilon}, t \in\right] 0, T[.
$$




\subsubsection{Two dimensional case}

Theorem 3.3. There exists $c>0$ independent on $\varepsilon$, verifying

$$
\left\|v_{\varepsilon}(x, t)-\frac{1}{\log (\varepsilon)} W(x, t)\right\|_{L^{2}\left(0, T ; H^{1}\left(\Omega_{z, \varepsilon}\right)\right.} \leq \frac{-c}{\log (\varepsilon)},
$$

where

$$
\left.W(x, t)=4 \pi v \sum_{j=1}^{2}\left[E^{j}(x-z) w_{0}(z, t)\right] e_{j}, \quad \forall(x, t) \in \Omega_{z, \varepsilon} \times\right] 0, T[,
$$

with $E^{j}(y)=E(y) e_{j}, 1 \leq j \leq 2,\left\{e_{j}\right\}_{j=1,2}$ is the $\mathbb{R}^{2}$ canonical basis and

$$
E(y)=\frac{1}{4 \pi v}\left(-\log (r) I+e_{r} e_{r}^{T}\right), \Pi(y)=\frac{y}{2 \pi r^{2}}, \quad \forall y \in \mathbb{R}^{2},
$$

represents the fundamental solution of the Stokes System in $\mathbb{R}^{2}$ with $r=\|y\|$ and $e_{r}=\frac{y}{r}$.

Using Theorem 3.3 it follows the velocity estimation in the perturbed fluid flow domain.

Corollary 3.4. We have

$$
v_{\varepsilon}(x, t)=W(x, t)+O\left(\frac{-1}{\log (\varepsilon)}\right), \quad x \in \Omega_{z, \varepsilon}, t>0
$$

\subsection{Asymptotic behavior of the shape function}

The topological sensitivity analysis for the non stationary Navier-Stokes operator in three and two dimensional cases is given in this section. The presented results are satisfied by all shape functions $j$ defined by (2) and $J_{\varepsilon}$ verifies the Assumption $(\mathcal{A})$.

\subsubsection{Three dimensional case}

Theorem 3.5. If $J_{\varepsilon}$ satisfies the Assumption $(\mathcal{A})$ with $\rho(\varepsilon)=\varepsilon$, then $j$ defined by (2) verifies

$$
j\left(\Omega \backslash \overline{\mathcal{O}_{z, \varepsilon}}\right)=j(\Omega)+\varepsilon\left[\int_{0}^{T} w_{0}(z, t) \cdot \mathcal{M}_{\mathcal{O}} u_{0}(z, t) d t+\delta \mathcal{J}\right]+o(\varepsilon),
$$

where

- $\quad$ the matrix $\mathcal{M}_{\mathcal{O}}$ is given by

$$
\mathcal{M}_{\mathcal{O}_{i j}}=\int_{\partial \mathcal{O}} \eta_{j}^{i}(y) d s(y), 1 \leq i, j \leq 3 .
$$

- $\quad u_{0}$ is the solution to the adjoint problem

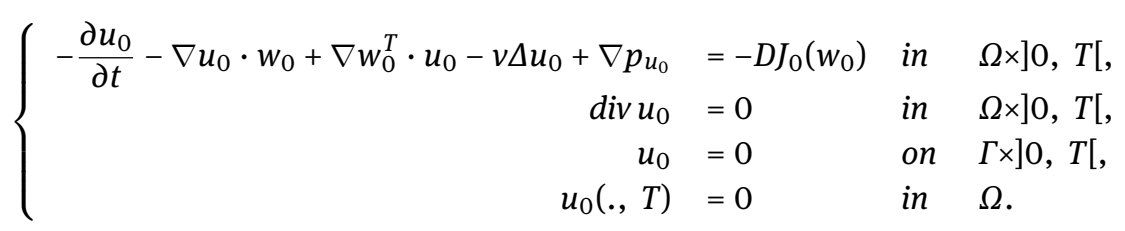


Corollary 3.6. If $\mathcal{O}=B(0,1)$ (the unit ball), $\eta_{j}(y)=-\frac{3 v}{2} e_{j}, \forall y \in \partial \mathcal{O}$ and

$$
j\left(\Omega \backslash \overline{\mathcal{O}_{z, \varepsilon}}\right)=j(\Omega)+\varepsilon\left[\int_{0}^{T} 6 \pi \nu w_{0}(z, t) \cdot u_{0}(z, t) d t+\delta \mathcal{J}\right]+o(\varepsilon) .
$$

\subsubsection{Two dimensional case}

Theorem 3.7. If $J_{\varepsilon}$ satisfies the Assumption ( $\mathcal{A}$ ) then $j$ defined by (2) verifies

$$
j\left(\Omega \backslash \overline{\mathcal{O}_{z, \varepsilon}}\right)=j(\Omega)+\frac{-1}{\log (\varepsilon)}\left[4 \pi v \int_{0}^{T} w_{0}(z, t) u_{0}(z, t) d t+\delta \mathcal{J}\right]+o\left(\frac{-1}{\log (\varepsilon)}\right),
$$

where $u_{0}$ is the adjoint state solution to the problem (9).

The proofs of Theorems 3.1, 3.3, 3.5 and 3.7 are relegated to section 5. The variation $\delta \mathcal{J}$ depends on the shape functions expressions. Some useful examples in numerical applications will be presented in section 3.3.

\subsection{Shape function examples}

\subsubsection{First example}

We define the shape function

$$
j\left(\Omega \backslash \overline{\mathcal{O}_{z, \varepsilon}}\right)=\int_{0}^{T} \int_{\Omega_{z, \varepsilon}}\left|w_{\varepsilon}-\mathcal{W}_{d}(., t)\right|^{2} d x d t
$$

where $\mathcal{W}_{d} \in L^{1}\left(0, T ; H^{1}(\Omega)\right)$ is a datum representing a desired fluid flow state.

This example concerns the $L^{2}$-norm shape function that has been used in geometric control problems like the optimization of location of some obstacle in a tank to approximate an object flow $\mathcal{W}_{d}$ (see [16]).

Proposition 3.8. The function

$$
J_{\varepsilon}(w)=\int_{\Omega_{z, \varepsilon}}\left|w-\mathcal{W}_{d}(., t)\right|^{2} d x, \quad \forall w \in H^{1}\left(\Omega_{z, \varepsilon}\right),
$$

satisfies the assumption $(\mathcal{A})$ with

$D J_{0}\left(w_{0}(., t)\right) v=2 \int_{\Omega}\left(w_{0}(., t)-\mathcal{W}_{d}(., t)\right) v d x, \quad \forall v \in H^{1}(\Omega)$,

$\delta \mathcal{J}(z)=0, \quad \forall z \in \Omega$.

\subsubsection{Second example}

We define the shape function which corresponds to the dissipation energy minimization

$$
j\left(\Omega \backslash \overline{\mathcal{O}_{z, \varepsilon}}\right)=\int_{0}^{T} \int_{\Omega_{z, \varepsilon}}\left|\nabla w_{\varepsilon}-\nabla \mathcal{W}_{d}(., t)\right|^{2} d x d t,
$$

where $\mathcal{W}_{d} \in L^{1}\left(0, T ; H^{2}(\Omega)\right)$ is a given datum. It was used in several optimization problems such as minimum drag problem [23], pipe bend design [10? ], cavity example [24], reconstruction of Tesla valve [13]. 
Proposition 3.9. The function

$$
J_{\varepsilon}(w)=\int_{\Omega_{z, \varepsilon}} v\left|\nabla w-\nabla \mathcal{W}_{d}(., t)\right|^{2} d x, \quad \forall w \in H^{1}\left(\Omega_{z, \varepsilon}\right),
$$

satisfies the assumption $(\mathcal{A})$ with

$$
\begin{aligned}
& D J_{0}\left(w_{0}(., t)\right) v=2 v \int_{\Omega}\left(\nabla w_{0}(., t)-\nabla \mathcal{W}_{d}(., t)\right) \nabla v d x, \quad \forall v \in H^{1}(\Omega),
\end{aligned}
$$

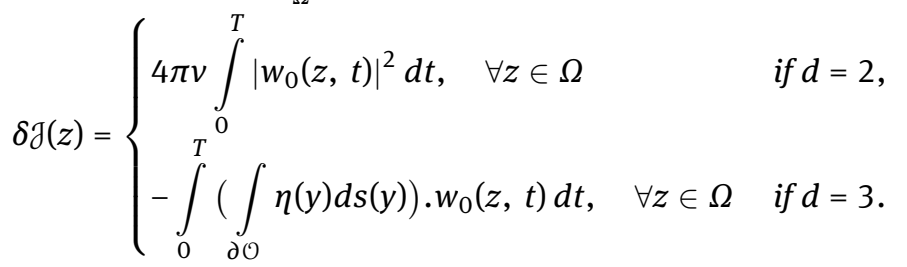

\section{Numerical results}

In this section, we deal with some numerical applications to validate the obtained theoretical results given in section 3.

\subsection{Validation of the asymptotic expansion}

To establish the numerical validation of Theorem 3.7, we consider the variation relating to $\varepsilon$ of

$$
\Delta_{z}(\varepsilon)=j\left(\Omega \backslash \overline{\mathcal{O}_{z, \varepsilon}}\right)-j(\Omega)+\frac{1}{\log (\varepsilon)} \delta j(z),
$$

where

$$
\delta j(z)=4 \pi v \int_{0}^{T} w_{0}(z, t) u_{0}(z, t) d t+\delta \mathcal{J}
$$

We expect to prove numerically that $\Delta_{z}(\varepsilon)$ satisfies the previously derived theoretical estimate $\Delta_{z}(\varepsilon)=$ $o\left(\frac{-1}{\log (\varepsilon)}\right)$.

To this aim, we consider the following data:

$-\Omega=] 0,1[\times] 0,1[$ is a square domain.

- The locations $z_{\varepsilon}^{i}=z_{i}+\varepsilon B(0,1)$ of the considered obstacles are arbitrary chosen (see Table 1).

- The shape function $j$ is defined by the semi-norm

$$
j\left(\Omega_{z, \varepsilon}\right)=\int_{0}^{T} \int_{\Omega_{z, \varepsilon}}\left|\nabla w_{\varepsilon}-\nabla \mathcal{W}_{d}\right|^{2} d x d t
$$

where $\mathcal{W}_{d}$ is a given velocity state.

Table 1: Location of obstacles

\begin{tabular}{lcccc}
\hline obstacle $z_{\varepsilon}^{i}$ & $z_{\varepsilon}^{1}$ & $z_{\varepsilon}^{2}$ & $z_{\varepsilon}^{3}$ & $z_{\varepsilon}^{4}$ \\
\hline location $z_{i}$ & $z_{1}=(0.2,0.8)$ & $z_{2}=(0.8,0.2)$ & $z_{3}=(0.5,0.5)$ & $z_{4}=(0.7,0.7)$ \\
\hline
\end{tabular}


In this case, the function $\Delta_{z_{i}}(\varepsilon)$ is defined by (see Theorem 3.7 and Proposition 3.9):

$$
\Delta_{z_{i}}(\varepsilon)=j\left(\Omega \backslash \overline{\mathcal{O}_{\varepsilon}^{i}}\right)-j(\Omega)+\frac{4 \pi v}{\log (\varepsilon)}\left(\int_{0}^{T} w_{0}\left(z_{i}, t\right) u_{0}\left(z_{i}, t\right) d t+\int_{0}^{T}\left|w_{0}\left(z_{i}, t\right)\right|^{2} d t\right) .
$$

The validation algorithm uses the following steps:

\section{The validation algorithm:}

- $\quad$ Step 1:

- compute the solution $w_{0}$ and the associated adjoint state $u_{0}$ in the domain $\Omega$.

- determine $j(\Omega)$ defined by (11).

- $\quad$ Step 2: For each obstacle $z_{\varepsilon}^{i}=z_{i}+\varepsilon B(0,1), i=1, \ldots, 4$ :

- determine the variation $\delta j\left(z_{i}\right)$ given in (10),

- choose $\varepsilon_{0}^{i}=\max \left\{\varepsilon>0\right.$, such that $\left.z_{i}+\varepsilon_{0}^{i} B(0,1) \subset \Omega\right\}$,

- compute an approximation of the function $\left.\left.\varepsilon \mapsto j\left(\Omega \backslash \overline{\mathcal{O}_{\varepsilon}^{i}}\right), \varepsilon \in\right] 0, \varepsilon_{0}^{i}\right]$.

- $\quad$ Step 3: Deduce numerically the function $\left.\left.\varepsilon \mapsto \log \left(\left|\Delta_{z^{i}}(\varepsilon)\right|\right), \varepsilon \in\right] 0, \varepsilon_{0}^{i}\right]$.

For each considered obstacle $\mathcal{O}_{\varepsilon}^{i}=z_{i}+\varepsilon B(0,1)$, we plot in Figure 2 the variation of $\log \left(\left|\Delta_{z_{i}}(\varepsilon)\right|\right)$ relating to $\log (-\log (\varepsilon))$.
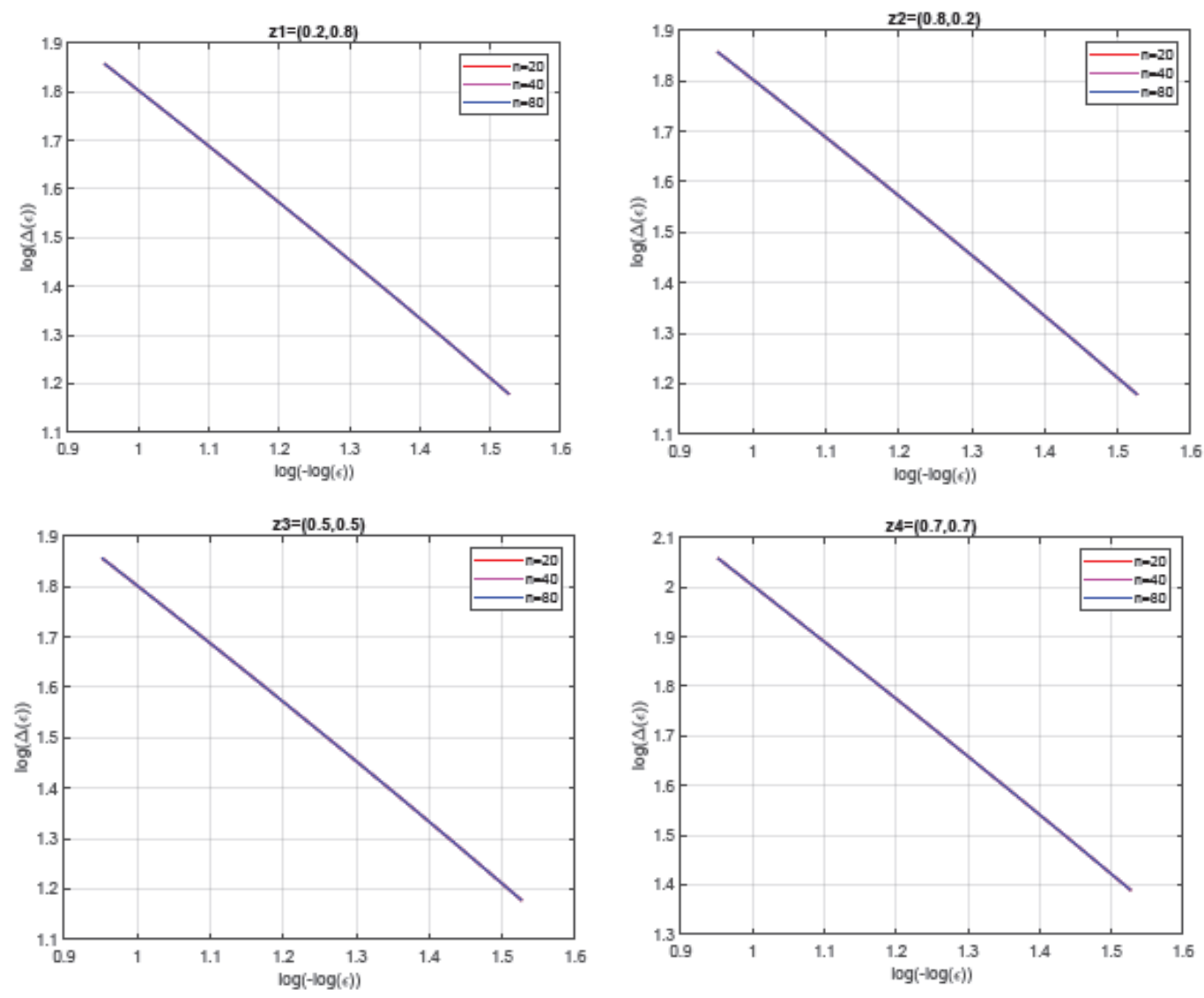

Fig. 2: Variation of $\log \left(\left|\Delta_{z_{i}}(\varepsilon)\right|\right)$ relating to $\log (-\log (\varepsilon))$. 
We define $\beta_{i}$ to describe the behavior of $\varepsilon \mapsto \Delta_{z_{i}}(\varepsilon)$ relating to $-\log (\varepsilon)$, i.e.

$$
\left|\Delta_{z_{i}}(\varepsilon)\right|=O\left((-\log (\varepsilon))^{\beta_{i}}\right) \text {. }
$$

It corresponds to the slope of the line approximating the variation $\varepsilon \mapsto \log \left(\left|\Delta_{z_{i}}(\varepsilon)\right|\right)$ relating to $\log (-\log (\varepsilon))$ for each obstacle $z_{\varepsilon}^{i}, i=1, \ldots, 4$.

From the plotted curves in Figure 2, one deduce the slopes $\beta_{i}, i=1, \ldots, 4$ in table 2.

Table 2: The obtained slopes $\beta_{i}$ of the lines associated with the obstacles $\mathcal{O}_{\varepsilon}^{i}, i=1, \ldots, 4$.

\begin{tabular}{ccccc}
\hline The considered obstacles $\mathcal{O}_{\varepsilon}^{i}$ & $\mathcal{O}_{\varepsilon}^{1}$ & $\mathcal{O}_{\varepsilon}^{2}$ & $\mathcal{O}_{\varepsilon}^{3}$ & $\mathcal{O}_{\varepsilon}^{4}$ \\
\hline The obtained slopes $\beta_{i}$ & -1.18 & -1.187 & -1.217 & -1.163 \\
\hline
\end{tabular}

We deduce that the numerical results confirm the behavior predicted by the theoretical estimate

$$
\Delta_{z_{i}}(\varepsilon)=o\left(\frac{-1}{\log (\varepsilon)}\right)
$$

\subsection{The Tesla micro-valve application}

The hydrocephalus treatment is a very important application in medicine. The problem is to optimize numerically the design of the 2D Tesla micro-valve at $\mathrm{Re}=100$. To solve this problem we consider the objective function as the forward energy dissipation and the diodicity as a constraint. The optimal domain is constructed through the insertion of some obstacles in the initial one. The problem leads to optimize the location of obstacles.

\subsubsection{Shape optimization problem}

We define $\Omega$ as the pentagon [15] having one inclined inlet $\Gamma_{\text {in }}$ and one horizontal outlet $\Gamma_{\text {out }}$ (see Figure 3). The aim is to find the fluid flow optimal domain $\Omega^{\star}$ which minimizes the dissipated energy by the forward

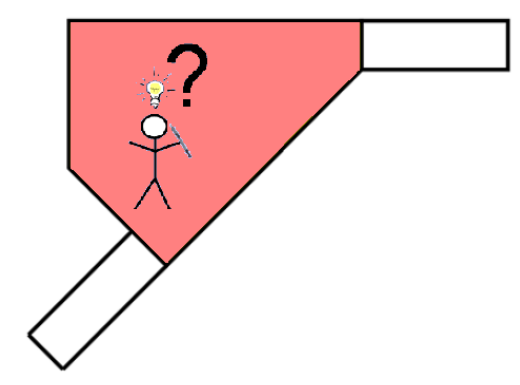

Fig. 3: Considered pentagon design domain

fluid flow and reproducing the original Tesla valve design given in Figure 1. This can be formulated :

$$
\left\{\begin{array}{l}
\text { Find } \Omega^{\star} \text { solution to } \\
\min _{\Omega \subset \mathcal{D}_{a d}} j(\Omega)
\end{array}\right.
$$


where

$$
\mathcal{D}_{a d}=\left\{D \subset \Omega \text { such that } \Gamma_{\text {in }} \subset \Gamma \cap \partial D, \Gamma_{\text {out }} \subset \Gamma \cap \partial D \text { and }|D| \leq V_{\text {desired }}\right\},
$$

with $|$.$| and V_{\text {desired }}$ represents respectively the Lebesgue measure and the target volume.

We recall that the performance of the Tesla valve is measured by diodicity $D i$ which is known as the ratio of the pressure drop in backward direction to that in forward direction, which is equivalent to the ratio of dissipation of reverse and forward flows [15]:

$$
D i=\frac{\Phi\left(w_{r}\right)}{\Phi\left(w_{f}\right)} \text { with } \Phi(w)=\int_{\Omega}\left[\frac{v}{2} \sum_{i, j}\left(\frac{\partial w_{i}}{\partial x_{j}}+\frac{\partial w_{j}}{\partial x_{i}}\right)^{2}\right],
$$

with $\left(w_{f}, p_{f}\right)$ and $\left(w_{r}, p_{r}\right)$ are respectively the solution to the Navier Stokes system in the forward and the reverse flows. Then, diodicity can be maximized by minimizing forward dissipation while maximizing reverse dissipation. That is why our optimization problem is defined with the diodicity $D i$ as a constraint; $D i>1$. Using the above definitions, the optimization problem [15] for reconstructing Tesla valve can be expressed as

* Objective: power dissipation of forward flow

$$
j(\Omega)=\Phi\left(w_{f}\right)=v \int_{0}^{T} \int_{\Omega}\left|\nabla w_{f}\right|^{2} d x d t,
$$

* Constraints

Volume fraction $\left|V_{\text {desired }}\right|<0.8\left|V_{0}\right|$.

- $\quad$ Diodicity $D i \geq c>1$.

- $\quad$ Navier Stokes equations for forward and backward directions.

We use the obtained theoretical results in 3.2.2 to solve (12).

\subsubsection{The topology optimization process}

To obtain the optimal domain, an iterative process is applied to construct a sequence of geometries $\left(\Omega_{k}\right)_{k \geq 0}$ with $\Omega_{0}=\Omega$ and $\Omega_{k+1}=\Omega_{k} \backslash \overline{\mathcal{O}_{k}}$ where $\mathcal{O}_{k}$ is an obstacle inserted in $\Omega_{k}$. To define the obstacle location and size, we find the function $\delta j_{k}$ defined by (see Theorem 3.7)

$$
\delta j_{k}(z)=4 \pi v\left[\int_{0}^{T}\left(w_{k}(z, t) u_{k}(z, t)+\left|w_{k}(z, t)\right|^{2}\right) d t\right], \forall z \in \Omega_{k},
$$

where

$-w_{k}$ represents the velocity, solution to the Navier-Stokes problem in $\Omega_{k}$

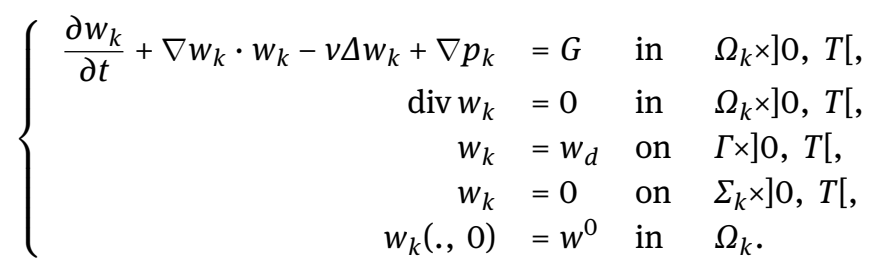

$-u_{k}$ is the adjoint state, solution to

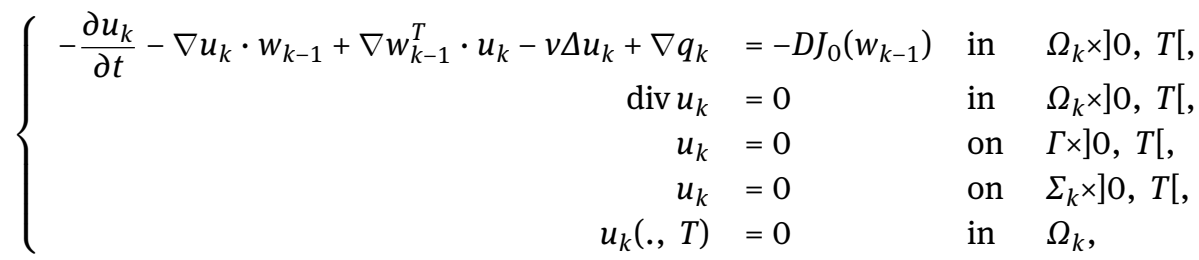


where $\Sigma_{k}=\partial\left(\cup_{l=0}^{k} \mathcal{O}_{l}\right)$ is the obstacle boundary inserted during the previous iterations.

The optimization steps are summarized as:

\section{The Algorithm:}

1. Initialization: Set $\Omega_{0}=\Omega$, and $k=0$

2. Repeat until $\left|\Omega_{k}\right| \leq V_{\text {desired }}$ :

(a) The topological sensitivity function:

- $\quad$ compute $w_{k}$, solution to the non stationary Stokes problem (14) in $\Omega_{k}$,

- compute $v_{k}$, solution to the associated adjoint problem (15) in $\Omega_{k}$,

- compute the term $\delta \mathcal{J}_{k}$ and deduce the function $\delta j_{k}(z), \forall z \in \Omega_{k}$.

(b) The obstacle to be inserted:

- determine $\rho_{k}^{\star} \in[0,1]$ such that $j\left(\Omega_{k} \backslash \overline{O_{\rho_{k}^{*}}^{k}}\right) \leq j\left(\Omega_{k} \backslash \overline{O_{\rho}^{k}}\right), \forall \rho \in[0,1]$,

- $\quad$ set $\mathcal{O}_{k}=\left\{x \in \Omega_{k} ; \delta j_{k}(x) \leq \rho_{k}^{\star} \delta_{\text {min }}^{k}\right\}$, where $\delta_{\text {min }}^{k}=\min \left(\delta j_{k}(z)\right)$.

(c) The new domain:

- $\quad$ set $\Omega_{k+1}=\Omega_{k} \backslash \overline{\mathcal{O}_{k}}$,

(d) $k \longleftarrow k+1$ and go to (2).

The stopping criteria is defined by the natural optimality condition

$$
\delta j^{k}(x) \geq 0, \forall x \in \Omega_{k} .
$$

This algorithm is like a descent method where $\delta j^{k}$ represents the descent direction and $\left|\mathcal{O}_{k}\right|=\left|\Omega_{k} \backslash \Omega_{k+1}\right|$ the step length. The parameter $\rho_{k}^{*}$ is chosen to allow $\rho \longmapsto j\left(\Omega_{k} \backslash \overline{O_{\rho}^{k}}\right)$ to decrease as much as possible. The computation of $\rho_{k}^{\star}$ in (b) can be viewed as line search step.

The numerical discretization of problems (14) and (15) is done by $P 1-b u b b l e / P 1$ finite element method [25]. The computation of the approximated solutions is achieved by the Uzawa's algorithm. The function $\delta j^{k}$ is computed piecewise constant over elements.

Next, we will apply the proposed algorithm to reconstruct Tesla micro valve.

\subsubsection{Reproducing the Tesla micro valve}

We illustrate in this section the strengths of topology optimization method, namely the ability to find optimal design using only information on boundary conditions and constraints without the need of initial design.

The considered design domain is the pentagon domain (see Figure 3). This problem example has already been studied by S. Lin and al. in [15] in the steady state regime using projection method.

For the forward direction, the inlet boundary velocity has a parabolic behavior ( $\mathrm{Re}=100$ relating to the inlet dimension). At the outlet boundary, the pressure is taken constant and no-slip condition is considered on the walls. For the backward flow direction, we reverse these boundary conditions. Besides, we prescribe solid regions close to the inlets/outlets to minimize the boundary effect on the final design solution. We illustrate the geometries obtained during the optimization process in Figure 4. The optimal domain is obtained after four iterations. It is nearly identical to literature $[1,15]$ (see Figure 5). 

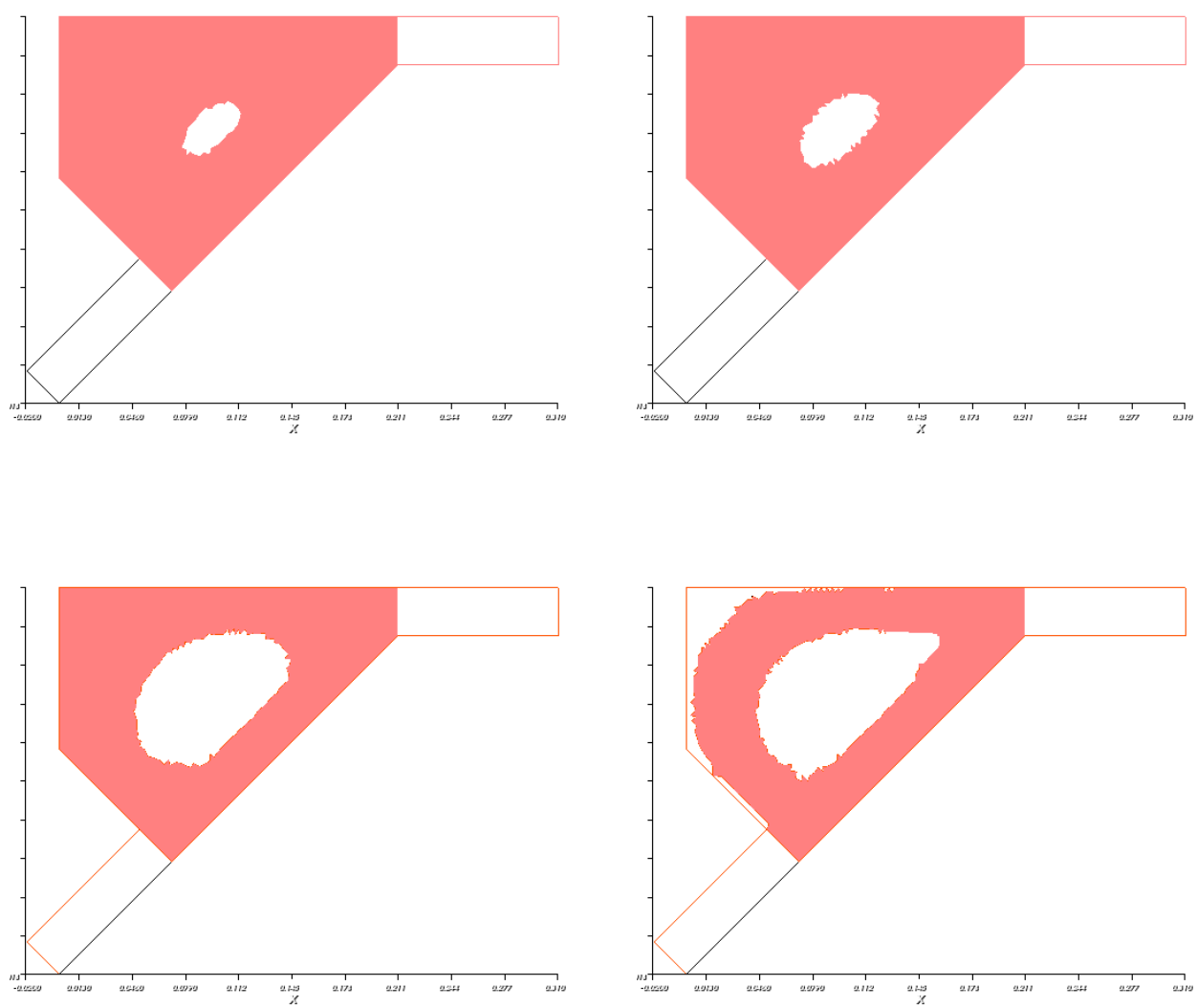

Fig. 4: Geometries obtained during the optimization process

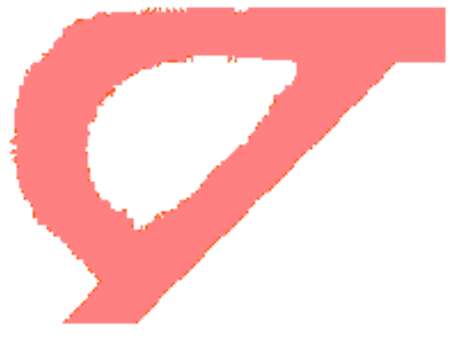

(a) mesh of obtained domain

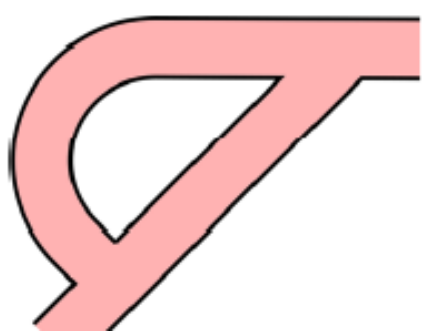

(b) Tesla-type valve

Fig. 5: mesh of obtained design (left) and reference Tesla valve (right)

\subsubsection{Discussion}

In the previous Figure, thanks to the topological gradient, we deduce an easy reconstruction of tesla valve. Now, we normalize the obtained tesla valve behavior by plotting the obtained forward and reverse flows respectively in figures 6(a) and 6(b). It is clear that the velocity field is strongly different for the two cases. 

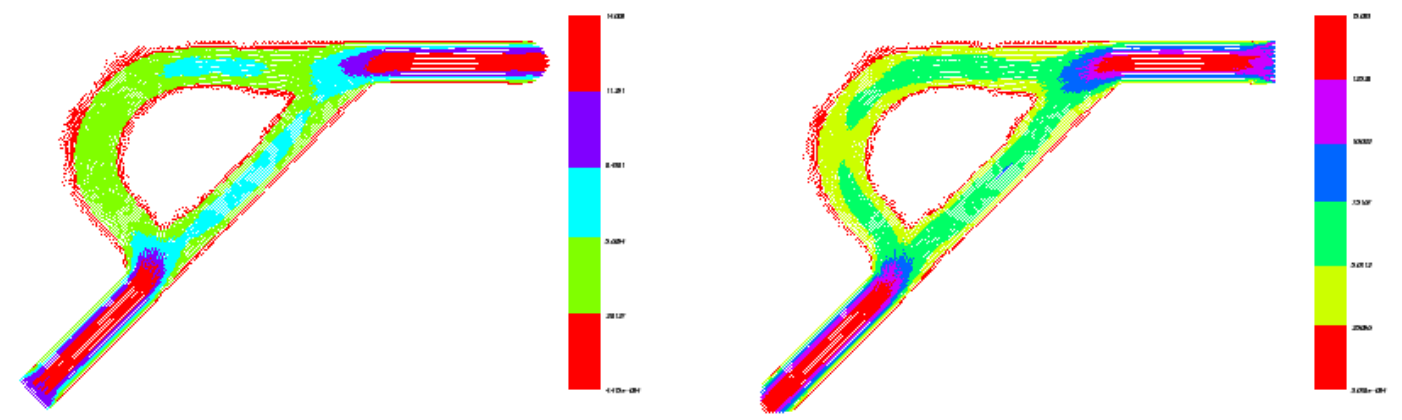

(a) Forward flow velocity field

(b) Backward flow velocity field

Fig. 6: Forward and backward flow velocity field

To study the obtained tesla valve performance, we calculate the diodicity. Using the energy view point expression of diodicity, the experimentally derived value is 1.137. In bibliography [26], the diodicity is well predicted using

$$
D i \cong 1+4.78 \text { * } 10^{-5} \star\left(N^{0.16} R e^{1.72}\right)
$$

with $N$ is the number of tesla valves and Re is the Reynolds number. Based on this expression, we found $D i \cong 1.1316$ which ensures an agreement between the obtained diodicity and the experimental one.

\section{Mathematical analysis}

This section deals with the proofs of Theorems 3.1, 3.3, 3.5 and 3.7.

\subsection{Proof of Theorem 3.1}

Let $Q$ be the pressure associated with the velocity $W$ :

$$
Q(x, t)=\frac{1}{\varepsilon} P\left(\frac{x-z}{\varepsilon}\right) \cdot w_{0}(z, t)=\frac{1}{\varepsilon} \sum_{j=1}^{3} P^{j}\left(\frac{x-z}{\varepsilon}\right) w_{0}^{j}(z, t),
$$

where $P^{j}$ is the pressure associated with the velocity $U^{j}$ solution to (6). Setting the variation

$$
z_{\varepsilon}=v_{\varepsilon}-W \text { and } p_{z_{\varepsilon}}=p_{v_{\varepsilon}}-Q .
$$

From (4) and (6), we can verify that $\left(z_{\varepsilon}, p_{z_{\varepsilon}}\right)$ is solution to

$$
\left\{\begin{aligned}
\frac{\partial z_{\varepsilon}}{\partial t} & -v \Delta z_{\varepsilon}+\nabla z_{\varepsilon} \cdot\left(w_{0}+W\right)+\nabla\left(w_{0}+W\right) \cdot z_{\varepsilon}+\nabla z_{\varepsilon} \cdot z_{\varepsilon}+\nabla p_{z_{\varepsilon}} & \\
& =-\frac{\partial W}{\partial t}-\nabla w_{0} \cdot W-\nabla W \cdot w_{0}-\nabla W \cdot W & \text { in } \left.\Omega_{z, \varepsilon} \times\right] 0, T[, \\
\operatorname{div} z_{\varepsilon} & =0 & \text { in } \left.\Omega_{z, \varepsilon} \times\right] 0, T[, \\
z_{\varepsilon} & =-W & \text { on } \Gamma \times] 0, T[, \\
z_{\varepsilon} & =-w_{0}(x, t)+w_{0}(z, t) & \text { on } \left.\partial \mathcal{O}_{\varepsilon} \times\right] 0, T[, \\
z_{\varepsilon}(.,, 0) & =0 & \text { in } \Omega_{z, \varepsilon} .
\end{aligned}\right.
$$


The last boundary condition follows due to the fact that $U^{j}=-e_{j}$ on $\partial \mathcal{O}$.

Moreover, since $\left|w_{0}\right|_{L^{2}\left(0, T ; H^{1}(\Omega)\right)}<v / k$, then $\varepsilon$ sufficiently small,

$$
\left|w_{0}+W\right|_{L^{2}\left(0, T ; H^{1}\left(\Omega_{z, \varepsilon}\right)\right)} \leq \alpha<v / k .
$$

Let $R>0$ such that $\overline{\mathcal{O}}_{z, \varepsilon} \subset B(z, R)$ and $\overline{B(z, R)} \subset \Omega$. Using the trace theorem, we obtain

$$
\begin{aligned}
\left\|z_{\varepsilon}\right\|_{L^{2}\left(0, T ; H^{1}\left(\Omega_{z, \varepsilon}\right)\right)} \leq c( & \left\|\frac{\partial W}{\partial t}\right\|_{L^{2}\left(0, T ; L^{2}\left(\Omega_{z, \varepsilon}\right)\right)} \\
& +\|W\|_{L^{2}\left(0, T ; H^{1}\left(\Omega_{R}\right)\right)}+\left\|w_{0}(x, t)-w_{0}(z, t)\right\|_{L^{2}\left(0, T ; L^{2}\left(\Omega_{z, \varepsilon}\right)\right)} \\
& \left.+\left\|\nabla w_{0} \cdot W+\nabla W \cdot w_{0}+\nabla W \cdot W\right\|_{L^{2}\left(0, T ; H^{-1}\left(\Omega_{z, \varepsilon}\right)\right)}\right),
\end{aligned}
$$

where $\Omega_{R}=\Omega \backslash \overline{B(z, R)}$.

Using (5) and the variable change $x=z+\varepsilon y$, we obtain

$$
\begin{aligned}
\left\|\frac{\partial W}{\partial t}\right\|_{L^{2}\left(0, T ; L^{2}\left(\Omega_{z, \varepsilon}\right)\right)} & =\left\|\frac{\partial w_{0}}{\partial t}(z, .)\right\|_{L^{2}(0, T)}\left\|U\left(\frac{x-z}{\varepsilon}\right)\right\|_{L^{2}\left(\Omega_{z, \varepsilon}\right)} \\
& =\varepsilon^{3 / 2}\left\|\frac{\partial w_{0}}{\partial t}(z, .)\right\|_{L^{2}(0, T)}\|U\|_{L^{2}\left(\left(\Omega_{z, \varepsilon}\right) / \varepsilon\right)} .
\end{aligned}
$$

By the same way, we have

$$
\begin{aligned}
\|W\|_{L^{2}\left(0, T ; H^{1}\left(\Omega_{R}\right)\right)} & \leq\left\|w_{0}(z, .)\right\|_{L^{2}(0, T)}\left(\left\|U\left(\frac{x-z}{\varepsilon}\right)\right\|_{L^{2}\left(\Omega_{R}\right)}+\left\|\nabla_{x} U\left(\frac{x-z}{\varepsilon}\right)\right\|_{L^{2}\left(\Omega_{R}\right)}\right), \\
& \leq\left\|w_{0}(z, .)\right\|_{L^{2}(0, T)}\left(\varepsilon^{3 / 2}\|U\|_{L^{2}\left(\left(\Omega_{R}\right) / \varepsilon\right)}+\varepsilon^{1 / 2}\left\|\nabla_{y} U\right\|_{\left.L^{2}\left(\Omega_{R}\right) / \varepsilon\right)}\right) .
\end{aligned}
$$

Using [19] (see also [27]), the velocity field $U^{j}$, solution to the exterior Stokes problem, satisfies the estimate

$$
\left\|U^{j}\right\|_{L^{2}\left(\left(\Omega_{R}\right) / \varepsilon\right)} \leq c \varepsilon^{-1 / 2} \text { and }\left\|\nabla_{y} U^{j}\right\|_{\left.L^{2}\left(\Omega_{R}\right) / \varepsilon\right)} \leq c \varepsilon^{1 / 2} .
$$

Then, using the smoothness of $w_{0}$ and the previous estimates, one can deduce

$$
\left\|\frac{\partial W}{\partial t}\right\|_{L^{2}\left(0, T ; L^{2}\left(\Omega_{z, \varepsilon}\right)\right)} \leq c \varepsilon \text { and }\|W\|_{L^{2}\left(0, T ; H^{1}\left(\Omega_{R}\right)\right)} \leq c \varepsilon .
$$

For the third term in (19). Expanding $w_{0}(x, t)=w_{0}(z, t)+\varepsilon \nabla w_{0}\left(\xi_{y}, t\right) y$ with $\xi_{y} \in \mathcal{O}_{z, \varepsilon}$ and using the fact that $\nabla w_{0}$ is uniformly bounded, it follows that

$$
\left\|w_{0}(x, t)-w_{0}(z, t)\right\|_{L^{2}\left(0, T ; L^{2}\left(\Omega_{z, \varepsilon}\right)\right)} \leq c \varepsilon .
$$

We now examine the last term in (19). Since $w_{0} \in L^{\infty}(\Omega)$,

$$
\begin{aligned}
\| \nabla w_{0} \cdot W+\nabla W \cdot w_{0} & +\nabla W \cdot W \|_{L^{2}\left(0, T ; H^{-1}\left(\Omega_{z, \varepsilon}\right)\right)} \leq c\left(\|W\|_{L^{2}\left(0, T ; H^{-1}\left(\Omega_{z, \varepsilon}\right)\right)}\right. \\
& +\|\nabla W\|_{L^{2}\left(0, T ; H^{-1}\left(\Omega_{z, \varepsilon}\right)\right)}+\|\nabla W \cdot W\|_{L^{2}\left(0, T ; H^{-1}\left(\Omega_{z, \varepsilon}\right)\right)}, \\
& \leq c\left(\|W\|_{L^{2}\left(0, T ; L^{2}\left(\Omega_{z, \varepsilon}\right)\right)}+|W|_{L^{2}\left(0, T ; H^{1}\left(\Omega_{z, \varepsilon}\right)\right)}\|W\|_{L^{2}\left(0, T ; H^{1}\left(\Omega_{z, \varepsilon}\right)\right)}\right),
\end{aligned}
$$

according to Lemma 4.2 in [17].

In addition, by Lemma 4.5 in [17], the variable change and the continuity of $w_{0}$, we can deduce

$$
\|W\|_{L^{2}\left(0, T ; L^{2}\left(\Omega_{z, \varepsilon}\right)\right)} \leq c \varepsilon, \quad|W|_{L^{2}\left(0, T ; H^{1}\left(\Omega_{z, \varepsilon}\right)\right)} \leq c \varepsilon^{1 / 2}
$$

and then

$$
\left\|\nabla w_{0} \cdot W+\nabla W \cdot w_{0}+\nabla W \cdot W\right\|_{L^{2}\left(0, T ; H^{-1}\left(\Omega_{z, \varepsilon}\right)\right)} \leq c \varepsilon .
$$

Finally, combining (20), (21) and (23) we deduce that

$$
\left\|z_{\varepsilon}\right\|_{L^{2}\left(0, T ; H^{1}\left(\Omega_{z, \varepsilon}\right)\right)} \leq c \varepsilon .
$$




\subsection{Proof of Theorem 3.3}

Let $Q$ be the pressure associated with the velocity $W$ :

$$
Q(x, t)=4 \pi v \Pi(x-z) \cdot w_{0}(z, t)=4 \pi v \sum_{j=1}^{2} \Pi^{j}(x-z) w_{0}^{j}(z, t),
$$

where $\Pi^{j}$ is the pressure associated with the velocity $E^{j}$.

Setting

$$
z_{\varepsilon}=v_{\varepsilon}-\frac{1}{\log (\varepsilon)} W \text { and } s_{\varepsilon}=p_{v_{\varepsilon}}-\frac{1}{\log (\varepsilon)} Q .
$$

From (1) and (3), we obtain that $\left(z_{\varepsilon}, s_{\varepsilon}\right)$ is solution to

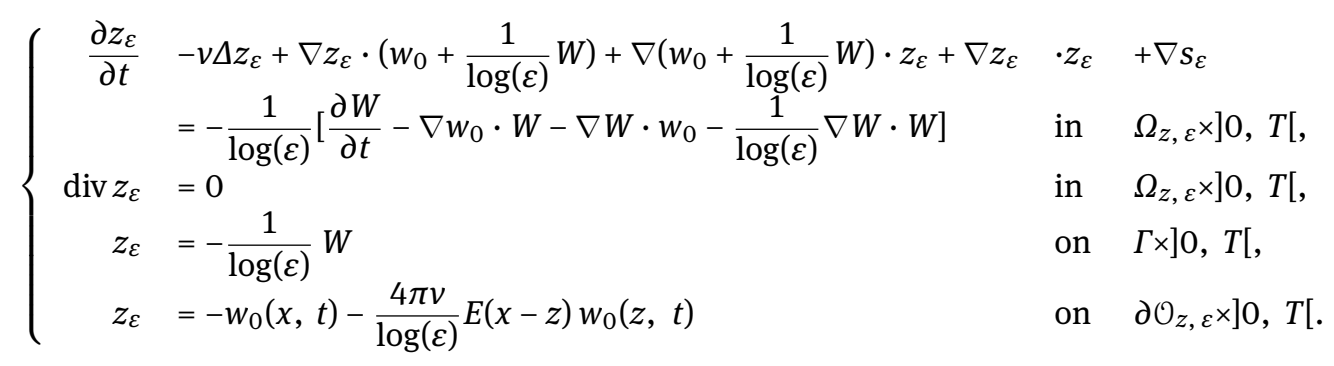

Using the relation $E((x-z) / \varepsilon)=E(x-z)+\frac{\log (\varepsilon)}{4 \pi v} I$, the last boundary condition can be rewritten as

$$
\left.z_{\varepsilon}=-w_{0}(x, t)+w_{0}(z, t)-\frac{4 \pi v}{\log (\varepsilon)} E((x-z) / \varepsilon) w_{0}(z, t) \text { on } \partial \mathcal{O}_{z, \varepsilon} \times\right] 0, T[.
$$

Then, by an energy inequality [28], it follows

$$
\begin{aligned}
& \left\|z_{\varepsilon}\right\|_{L^{2}\left(0, T ; H^{1}\left(\Omega_{z, \varepsilon}\right)\right)} \\
& \leq \frac{-c}{\log (\varepsilon)}\left[\left\|\frac{\partial W}{\partial t}\right\|_{L^{2}\left(0, T ; L^{2}\left(\Omega_{z, \varepsilon}\right)\right)}+\|W\|_{L^{2}\left(0, T ; H^{1 / 2}(\Gamma)\right)}\right. \\
& +\log (\varepsilon)\left\|w_{0}(z+\varepsilon y, t)-w_{0}(z, t)\right\|_{L^{2}\left(0, T ; H^{1 / 2}\left(\partial \mathcal{O}_{z, \varepsilon}\right)\right)} \\
& +4 \pi v\left\|E((x-z) / \varepsilon) w_{0}(z, t)\right\|_{L^{2}\left(0, T ; H^{1 / 2}\left(\partial \mathcal{O}_{z, \varepsilon}\right)\right)} \\
& \left.+\left\|\nabla w_{0} \cdot W+\nabla W \cdot w_{0}+\frac{1}{\log (\varepsilon)} \nabla W \cdot W\right\|_{L^{2}\left(0, T ; L^{2}\left(\Omega_{z, \varepsilon}\right)\right)}\right] .
\end{aligned}
$$

We estimate in the following each term in (26) separately.

We remark that:

- Since $\mathcal{O}$ is an open domain containing the origin, $\exists r>0$ such that $\overline{B(0, r)} \subset \mathcal{O}$.

- $\Omega$ is a bounded domain in such a way that $\exists R>0$ such that $\bar{\Omega} \subset B(z, R), \forall z \in \Omega$.

- We have $\Omega_{z, \varepsilon}-z=\left\{x-z, x \in \Omega_{z, \varepsilon}\right\} \subset C(0, r \varepsilon, R)=\left\{y \in \mathbb{R}^{2} ; r \varepsilon<|y|<R\right\}$.

From the fact that $C(0, r \varepsilon, R) \subset \mathbb{R}^{2} \backslash 0$, it follows that the function $\psi: y \mapsto \log (|y|)$ is smooth in $C(0, r \varepsilon, R)$ and we have $\|\psi\|_{0, C(0, r \varepsilon, R)} \leq c$. Then, using the cylindrical coordinate system, one can prove that $\exists c>0$, independent of $\varepsilon$, such that

$$
\begin{array}{cc}
\|E(x-z)\|_{L^{2}\left(0, T ; L^{2}\left(\Omega_{z, \varepsilon}\right)\right)} & \leq\|E(y)\|_{C(0, r \varepsilon, R)} \leq c, \\
\|\nabla E(x-z)\|_{L^{2}\left(0, T ; L^{2}\left(\Omega_{z, \varepsilon}\right)\right.} & \leq c \sqrt{-\log (\varepsilon)} .
\end{array}
$$

- Estimate of the first term in (26): Using that $w_{0} \in H^{1}\left(0, T ; H^{1}(\Omega)\right)$, we obtain

$$
\left\|\frac{\partial W}{\partial t}\right\|_{L^{2}\left(0, T ; L^{2}\left(\Omega_{z, \varepsilon}\right)\right)}=4 \pi v\left\|\frac{\partial w_{0}}{\partial t}(z, t)\right\|_{L^{2}(0, T)}\|E(x-z)\|_{L^{2}\left(\Omega_{z, \varepsilon}\right)}=O(1) .
$$

- Estimate of the last term of (26): 
Since $w_{0}$ and $\nabla w_{0}$ belong to $L^{\infty}(\Omega)$, we have

$$
\begin{aligned}
& \left\|\nabla w_{0} \cdot W+\nabla W \cdot W_{0}+\frac{1}{\log (\varepsilon)} \nabla W \cdot W\right\|_{L^{2}\left(0, T ; L^{2}\left(\Omega_{z, \varepsilon}\right)\right)} \leq \\
& c\left(\|W\|_{L^{2}\left(0, T ; L^{2}\left(\Omega_{z, \varepsilon}\right)\right)}+\|\nabla W\|_{L^{2}\left(0, T ; L^{2}\left(\Omega_{z, \varepsilon}\right)\right)}+\frac{1}{\log (\varepsilon)}\|\nabla W\|_{L^{2}\left(0, T ; L^{2}\left(\Omega_{z, \varepsilon}\right)\right)}\|W\|_{L^{2}\left(0, T ; L^{2}\left(\Omega_{z, \varepsilon}\right)\right)}\right) .
\end{aligned}
$$

Using the definition of $W$, we can deduce the following estimates

$$
\|W\|_{L^{2}\left(0, T ; L^{2}\left(\Omega_{z, \varepsilon}\right)\right)} \leq c, \quad\|\nabla W\|_{L^{2}\left(0, T ; L^{2}\left(\Omega_{z, \varepsilon}\right)\right)} \leq c \sqrt{-\log (\varepsilon)} .
$$

Yet, we have

$$
\left\|\nabla w_{0} \cdot W+\nabla W \cdot w_{0}+\frac{1}{\log (\varepsilon)} \nabla W \cdot W\right\|_{L^{2}\left(0, T ; L^{2}\left(\Omega_{z, \varepsilon}\right)\right)} \leq \quad c \sqrt{-\log (\varepsilon)} .
$$

- Estimate of boundary condition imposed on $\Gamma$ :

Let $\widetilde{R}>0$ such that $\mathcal{O}_{z, \varepsilon} \subset B(z, \widetilde{R})$ and $\overline{B(z, \widetilde{R})} \subset \Omega$. Since $z \notin \Omega_{\widetilde{R}}=\Omega \backslash \overline{B(z, \widetilde{R})}$, the function $x \mapsto E(x-z)$ belongs to $\mathcal{C}^{1}\left(\Omega_{\widetilde{R}}\right)$. By the trace theorem, we have

$$
\begin{aligned}
\|W\|_{L^{2}\left(0, T ; H^{1 / 2}(\Gamma)\right.} & =4 \pi v\left\|w_{0}(z, t)\right\|_{L^{2}(0, T)}\|E(x-z)\|_{H^{1 / 2}(\Gamma)} \\
& \leq 4 \pi v\left\|w_{0}(z, t)\right\|_{L^{2}(0, T)}\left[\|E(x-z)\|_{L^{2}\left(\Omega_{R}\right)}+\|\nabla E(x-z)\|_{L^{2}\left(\Omega_{R}\right)}\right] .
\end{aligned}
$$

Therefore, $\|W\|_{L^{2}\left(0, T ; H^{1 / 2}(\Gamma)\right.}$ is uniformly bounded with respect to $\varepsilon$.

- Estimate of boundary condition imposed on $\partial \mathcal{O}_{z, \varepsilon}$ :

Using the theorem of trace and the smoothness of $w_{0}$ in $\left.\mathcal{O}_{z, \varepsilon} \times\right] 0, T$, one can obtain

$$
\left\|w_{0}(x, t)-w_{0}(z, t)\right\|_{L^{2}\left(0, T ; H^{1 / 2}\left(\partial \mathcal{O}_{z, \varepsilon}\right)\right)} \leq c \varepsilon .
$$

Then, the first boundary term on $\partial \mathcal{O}_{z, \varepsilon}$ satisfies

$$
\log (\varepsilon)\left\|w_{0}(x, t)-w_{0}(z, t)\right\|_{L^{2}\left(0, T ; H^{1 / 2}\left(\partial \mathcal{O}_{z, \varepsilon}\right)\right)}=o\left(\frac{-1}{\log (\varepsilon)}\right) .
$$

To estimate the last boundary term, we use that $\mathcal{O}$ contains the origin.

Setting $\mathcal{O}_{r}=\mathcal{O} \backslash \overline{B(0, r)}$ and $\mathcal{O}_{r, \varepsilon}=z+\varepsilon \mathcal{O}_{r}$. Using the theorem of trace and the variable change $x=z+\varepsilon y$, we obtain

$$
\begin{aligned}
\| E((x-z) / \varepsilon) & w_{0}(z, t) \|_{L^{2}\left(0, T ; H^{1 / 2}\left(\partial O_{z, \varepsilon}\right)\right)} \\
& \leq\left\|w_{0}(z, t)\right\|_{L^{2}(0, T)}\left(\|E((x-z) / \varepsilon)\|_{L^{2}\left(\mathcal{O}_{r, \varepsilon}\right)}+\left\|\nabla_{x} E((x-z) / \varepsilon)\right\|_{L^{2}\left(\mathcal{O}_{r, \varepsilon}\right)}\right) \\
& \leq\left\|w_{0}(z, t)\right\|_{L^{2}(0, T)}\left(\varepsilon\|E(y)\|_{L^{2}\left(\mathcal{O}_{r}\right)}+\varepsilon^{1 / 2}\left\|\nabla_{y} E(y)\right\|_{L^{2}\left(\mathcal{O}_{r}\right)}\right) .
\end{aligned}
$$

From the fact that $y \mapsto E(y)$ is sufficiently smooth in $\mathcal{O}_{r} \subset \mathbb{R}^{2} \backslash\{0\}$, the last quantity is uniformly bounded and then

$$
\frac{-4 \pi v}{\log (\varepsilon)}\left\|E((x-z) / \varepsilon) w_{0}(z, t)\right\|_{L^{2}\left(0, T ; H^{1 / 2}\left(\partial O_{z, \varepsilon}\right)\right)} \leq \frac{-c \varepsilon^{1 / 2}}{\log (\varepsilon)} .
$$

Finally, combining the above estimates, we obtain, $\exists c>0$, independent of $\varepsilon$, such as

$$
\left\|z_{\varepsilon}\right\|_{L^{2}\left(0, T ; H^{1}\left(\Omega_{z, \varepsilon}\right)\right)} \leq \frac{-c}{\log (\varepsilon)}
$$

which ends the proof of Theorem 3.3. 


\subsection{Asymptotic analysis}

This section deals with the proofs of the Theorems presented in paragraphs 3.2 and 3.3.

Using the assumption $(\mathcal{A})$,

$$
\begin{aligned}
j\left(\Omega \backslash \overline{\mathcal{O}_{z, \varepsilon}}\right)-j(\Omega) & =\int_{0}^{T} J_{\varepsilon}\left(w_{\varepsilon}(., t)\right) d t-\int_{0}^{T} J_{0}\left(w_{0}(., t)\right) d t \\
& =\int_{0}^{T} D J_{0}\left(w_{0}(., t)\right)\left(w_{\varepsilon}(., t)-w_{0}(., t)\right) d t+\rho(\varepsilon) \delta \mathcal{J}+o(\rho(\varepsilon)),
\end{aligned}
$$

where $w_{\varepsilon}$ is extended by zero inside the domain $\mathcal{O}_{z, \varepsilon}$.

Using Green formula and that $w_{\varepsilon}=0$ in $\mathcal{O}_{\varepsilon}$, it follows

$$
\begin{aligned}
j\left(\Omega \backslash \overline{\mathcal{O}_{z, \varepsilon}}\right)-j(\Omega)= & -v \int_{0}^{T} \int_{\Omega_{z, \varepsilon}} \nabla v_{\varepsilon} \nabla u_{0} d x d t-\int_{0}^{T} \int_{\Omega_{z, \varepsilon}} \frac{\partial v_{\varepsilon}}{\partial t} u_{0} d x d t+\int_{0}^{T} \int_{\mathcal{O}_{z, \varepsilon}} \frac{\partial w_{0}}{\partial t} u_{0} d x d t \\
& +v \int_{0}^{T} \int_{\mathcal{O}_{z, \varepsilon}} \nabla w_{0} \nabla u_{0} d x d t-\int_{0 \Omega_{z, \varepsilon}}\left(\nabla v_{\varepsilon} w_{0}+\nabla w_{0} v_{\varepsilon}\right) u_{0} d x d t \\
& +2 \int_{0}^{T} \int_{\mathcal{O}_{z, \varepsilon}}\left(\nabla w_{0} w_{0}\right) u_{0} d x d t+\rho(\varepsilon) \delta \mathcal{J}+o(\rho(\varepsilon)),
\end{aligned}
$$

where $u_{0}$ is the solution to the associated adjoint problem.

From (4) and the fact that $w_{0}=0$ on $\left.\Gamma \times\right] 0, T[$, we obtain

$$
\begin{gathered}
-v \int_{\mathbb{Q}_{z, \varepsilon}}^{T} \nabla v_{\varepsilon} \nabla u_{0} d x d t-\int_{0}^{T} \int_{\Omega_{z, \varepsilon}} \frac{\partial v_{\varepsilon}}{\partial t} u_{0} d x d t-\int_{0}^{T} \int_{\Omega_{z, \varepsilon}}\left(\nabla v_{\varepsilon} w_{0}+\nabla w_{0} v_{\varepsilon}\right) u_{0} d x d t \\
=-\int_{0}^{T} \int_{\partial \mathcal{O}_{z, \varepsilon}} \sigma\left(v_{\varepsilon}, p_{v_{\varepsilon}}\right) n u_{0} d s d t+\int_{0}^{T} \int_{\Omega_{z, \varepsilon}}\left(\nabla v_{\varepsilon} v_{\varepsilon}\right) u_{0} d x d t .
\end{gathered}
$$

Therefore,

$$
\begin{aligned}
& j\left(\Omega \backslash \overline{\mathcal{O}_{z, \varepsilon}}\right)-j(\Omega) \\
& =\int_{0}^{T} \int_{\mathcal{O}_{z, \varepsilon}} \frac{\partial w_{0}}{\partial t} u_{0} d x d t+v \int_{0}^{T} \int_{\mathcal{O}_{z, \varepsilon}}^{T} \nabla w_{0} \nabla u_{0} d x d t+2 \int_{0}^{T} \int_{\mathcal{O}_{z, \varepsilon}}\left(\nabla w_{0} w_{0}\right) u_{0} d x d t \\
& -\int_{0}^{T} \int_{\partial \mathcal{O}_{z, \varepsilon}} \sigma\left(v_{\varepsilon}, p_{v_{\varepsilon}}\right) n u_{0} d s d t+\int_{0}^{T} \int_{\Omega_{z, \varepsilon}}\left(\nabla v_{\varepsilon} v_{\varepsilon}\right) u_{0} d x d t+\rho(\varepsilon) \delta \mathcal{J}(z)+o(\rho(\varepsilon)) .
\end{aligned}
$$

We begin by giving the estimate of the first three terms in (33).

Lemma 5.1. The integral terms in (33) satisfy the estimate

$$
\int_{0}^{T} \int_{\mathcal{O}_{z, \varepsilon}} \frac{\partial w_{0}}{\partial t} u_{0} d x d t+v \int_{0}^{T} \int_{\mathcal{O}_{z, \varepsilon}} \nabla w_{0} \nabla u_{0} d x d t+2 \int_{0}^{T} \int_{\mathcal{O}_{z, \varepsilon}}\left(\nabla w_{0} w_{0}\right) u_{0} d x d t=O\left(\varepsilon^{d}\right) .
$$


Proof 5.2. Using the variable change $x=z+\varepsilon y$, the first integral term in (33) can be written

$$
\begin{aligned}
\int_{0}^{T} \int_{\mathcal{O}_{z, \varepsilon}} \frac{\partial w_{0}}{\partial t} u_{0} d x d t & =\varepsilon^{d} \int_{0}^{T} \int_{\mathcal{O}}\left(\frac{\partial w_{0}(z+\varepsilon y, t)}{\partial t} u_{0}(z+\varepsilon y, t)-\frac{\partial w_{0}(z, t)}{\partial t} u_{0}(z, t) d y d t\right. \\
& +\varepsilon^{d}|\mathcal{O}| \int_{0}^{T} \frac{\partial w_{0}(z, t)}{\partial t} u_{0}(z, t) d t
\end{aligned}
$$

where $|\mathcal{O}|$ denotes the Lebesgue measure of $\mathcal{O}$.

Using that $w_{0}$ and $u_{0}$ are smooth near $z$, one can deduce that

$$
\int_{0}^{T} \int_{\mathcal{O}_{z, \varepsilon}}^{T} \frac{\partial w_{0}}{\partial t} u_{0} d x d t+v \int_{0}^{T} \int_{\mathcal{O}_{z, \varepsilon}} \nabla w_{0} \nabla u_{0} d x d t+2 \int_{0}^{T} \int_{\mathcal{O}_{z, \varepsilon}}\left(\nabla w_{0} w_{0}\right) u_{0} d x d t=O\left(\varepsilon^{d}\right) .
$$

By the same arguments, we can estimate the two other terms in (33).

The shape function variation can be rewritten

$$
j\left(\Omega \backslash \overline{\mathcal{O}_{z, \varepsilon}}\right)-j(\Omega)=-\int_{0}^{T} \int_{\partial \mathcal{O}_{z, \varepsilon}} \sigma\left(v_{\varepsilon}, p_{v_{\varepsilon}}\right) n u_{0} d s d t+\int_{0}^{T} \int_{\Omega_{z, \varepsilon}}\left(\nabla v_{\varepsilon} v_{\varepsilon}\right) u_{0} d x d t+\rho(\varepsilon) \delta \mathcal{J}(z)+o(\rho(\varepsilon)) .
$$

We are now ready to prove the established results in Theorems 3.5 and 3.7 and propositions 3.8 and 3.9.

\subsubsection{Proof of Theorem 3.5}

Using an integration by parts and the fact that $\operatorname{div}\left(v_{\varepsilon}\right)=0$ yield

$$
\begin{aligned}
\left|\int_{0 \Omega_{z, \varepsilon}}^{T}\left(\nabla v_{\varepsilon} v_{\varepsilon}\right) u_{0} d x d t\right| & =\left|-\int_{0 \Omega_{z, \varepsilon}}^{T}\left(\nabla\left(u_{0}\right) \cdot\left(v_{\varepsilon}\right)\right) \cdot v_{\varepsilon} d x d t\right| \\
& \leq\left\|\nabla u_{0}\right\|_{L^{\infty}\left(\Omega_{z, \varepsilon}\right)}\left\|v_{\varepsilon}\right\|_{L^{2}\left(\Omega_{z, \varepsilon}\right)}^{2} \\
& \leq 2\left\|\nabla u_{0}\right\|_{L^{\infty}\left(\Omega_{z, \varepsilon}\right)}\left(\left\|z_{\varepsilon}\right\|_{L^{2}\left(\Omega_{z, \varepsilon}\right)}^{2}+\|W\|_{L^{2}\left(\Omega_{z, \varepsilon}\right)}^{2}\right) \\
& \leq c \varepsilon^{2} .
\end{aligned}
$$

Then, the shape function variation can be written

$$
j\left(\Omega \backslash \overline{\mathcal{O}_{z, \varepsilon}}\right)-j(\Omega)=-\int_{0}^{T} \int_{\partial \mathcal{O}_{z, \varepsilon}} \sigma\left(v_{\varepsilon}, p_{v_{\varepsilon}}\right) n u_{0} d s d t+\varepsilon \delta \mathcal{J}(z)+o(\varepsilon) .
$$

From the definition of $\left(z_{\varepsilon}, s_{\varepsilon}\right)$ and the variable change $x=z+\varepsilon y$, we have

$$
\begin{aligned}
\int_{0 \partial \mathcal{O}_{z, \varepsilon}}^{T} \sigma\left(v_{\varepsilon}, p_{v_{\varepsilon}}\right) n u_{0} d s d t & =\int_{0}^{T} \int_{\partial \mathcal{O}_{z, \varepsilon}} \sigma\left(z_{\varepsilon}, s_{\varepsilon}\right) n u_{0} d s d t \\
& +\varepsilon \int_{0}^{T} w_{0}(z, t) \cdot\left(\int_{\partial \mathcal{O}} \sigma(U, P)(y) n(y) u_{0}(z+\varepsilon y, t) d s(y)\right) d t,
\end{aligned}
$$

where $\sigma(U, P) n$ is the $3 \times 3$ matrix defined by

$$
(\sigma(U, P) n)_{i j}=\left(\sigma\left(U^{j}, P^{j}\right)(y) n(y)\right)_{i}, 1 \leq i, j \leq 3 .
$$


By the trace theorem, Theorem 3.1 and that $u_{0}$ is smooth in $\mathcal{O}_{z, \varepsilon}$,

$$
\left|\int_{0}^{T} \int_{\partial \mathcal{O}_{z, \varepsilon}} \sigma\left(z_{\varepsilon}, s_{\varepsilon}\right) n u_{0} d s d t\right| \leq\left\|\sigma\left(z_{\varepsilon}, s_{\varepsilon}\right) n\right\|_{L^{2}\left(0, T ; H^{-1 / 2}\left(\partial \mathcal{O}_{z, \varepsilon}\right)\right)}\left\|u_{0}\right\|_{L^{2}\left(0, T ; H^{1}\left(\mathcal{O}_{z, \varepsilon}\right)\right)}=o(\varepsilon) .
$$

Making the variable change $x=z+\varepsilon y$, expanding $u_{0}(z+\varepsilon y, t)=u_{0}(z, t)+\varepsilon \nabla u_{0}\left(\xi_{y}, t\right) y$ with $\xi_{y} \in \mathcal{O}_{z, \varepsilon}$ and using that $\nabla u_{0}$ is uniformly bounded, we obtain

$$
\begin{aligned}
\int_{0}^{T} & \int_{\partial \mathcal{O}_{z, \varepsilon}} \sigma\left(v_{\varepsilon}, p_{v_{\varepsilon}}\right) n u_{0} d s d t \\
\quad & \varepsilon \int_{0}^{T} w_{0}(z, t) \cdot\left(\int_{\partial \mathcal{O}} \sigma(U, P)(y) n d s(y)\right) u_{0}(z, t) d t \\
& +\varepsilon \int_{0}^{T} w_{0}(z, t)\left(\int_{\partial \mathcal{O}} \sigma(U, P)(y) n(y)\left[u_{0}(z+\varepsilon y, t)-u_{0}(z, t)\right] d s(y)\right) d t+o(\varepsilon) .
\end{aligned}
$$

Due to the jump condition of the single layer potential $\sigma\left(U^{j}, P^{j}\right) n=-\eta^{j}+\sigma\left(V^{j}, S^{j}\right) n$, where $\left(V^{j}, S^{j}\right)$ is the solution to the interior problem

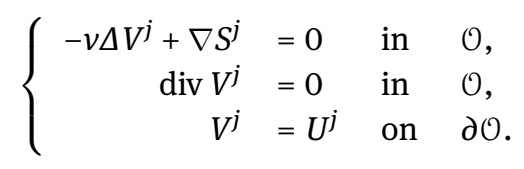

By the fact that $\operatorname{div} \sigma\left(V^{j}, S^{j}\right)=v \Delta V^{j}-\nabla S^{j}=0$ in $\mathcal{O}$, we have $\int_{\partial \mathcal{O}} \sigma\left(V^{j}, S^{j}\right)(y) n d s=0$.

Then, we obtain

$$
\int_{0}^{T} \int_{\partial \mathcal{O}_{z, \varepsilon}} \sigma\left(v_{\varepsilon}, p_{v_{\varepsilon}}\right) n u_{0} d s d t=-\varepsilon \int_{0}^{T} w_{0}(z, t) .\left(\int_{\partial \mathcal{O}} \eta(y) d s(y) u_{0}(z, t)\right) d t+o(\varepsilon) .
$$

Consequently, the shape function $j$ admits the asymptotic expansion

$$
j\left(\Omega \backslash \overline{\mathcal{O}_{z, \varepsilon}}\right)=j(\Omega)+\varepsilon\left[\int_{0}^{T} w_{0}(z, t) \cdot \mathcal{M}_{\mathcal{O}} u_{0}(z, t) d t+\delta \mathcal{J}\right]+o(\varepsilon),
$$

where $\mathcal{M}_{\mathcal{O}}$ is the matrix given by

$$
\mathcal{M}_{\mathcal{O} i j}=-\int_{\partial \mathcal{O}} \eta_{j}^{i}(y) d s(y), 1 \leq i, j \leq 3
$$

\subsubsection{Proof of Theorem 3.7}

The shape function variation is given by

$$
j\left(\Omega \backslash \overline{\mathcal{O}_{z, \varepsilon}}\right)-j(\Omega)=-\int_{0}^{T} \int_{\partial \mathcal{O}_{z, \varepsilon}} \sigma\left(v_{\varepsilon}, p_{v_{\varepsilon}}\right) n u_{0} d s d t+\int_{0}^{T} \int_{\Omega_{z, \varepsilon}}\left(\nabla v_{\varepsilon} v_{\varepsilon}\right) u_{0} d x d t+\frac{-1}{\log (\varepsilon)} \delta \mathcal{J}(z)+o\left(\frac{-1}{\log (\varepsilon)}\right) .
$$

Recall that the term $(W, Q)$ describing the perturbation due to the presence of a small obstacle $\mathcal{O}_{z, \varepsilon}$ is given by: $\left.\forall(x, t) \in \Omega_{z, \varepsilon} \times\right] 0, T[$,

$$
W(x, t)=4 \pi v \sum_{j=1}^{2}\left[E^{j}(x-z) w_{0}(z, t)\right] e_{j}, Q(x, t)=4 \pi v \sum_{j=1}^{2} \Pi^{j}(x-z) w_{0}^{j}(z, t),
$$


where $E^{j}(y)=E(y) e_{j}$ and $\Pi^{j}(y)=\Pi(y) \cdot e_{j}, 1 \leq j \leq 2$.

Applying an integration by parts and using the fact that $\operatorname{div}\left(v_{\varepsilon}\right)=0$ provides

$$
\int_{0}^{T} \int_{\Omega_{z, \varepsilon}}\left(\nabla v_{\varepsilon} v_{\varepsilon}\right) u_{0} d x d t=-\int_{0}^{T} \int_{\Omega_{z, \varepsilon}}\left(\nabla u_{0} \cdot v_{\varepsilon}\right) \cdot v_{\varepsilon} d x d t
$$

Then,

It follows that

$$
\begin{aligned}
\left|\int_{0} \int_{\Omega_{z, \varepsilon}}\left(\nabla v_{\varepsilon} v_{\varepsilon}\right) u_{0} d x d t\right| & \leq\left\|\nabla u_{0}\right\|_{L^{\infty}\left(\Omega_{z, \varepsilon}\right)}\left\|v_{\varepsilon}\right\|_{L^{2}\left(\Omega_{z, \varepsilon}\right)}^{2} \\
& \leq 2\left\|\nabla u_{0}\right\|_{L^{\infty}\left(\Omega_{z, \varepsilon}\right)}\left[\left\|z_{\varepsilon}\right\|_{L^{2}\left(\Omega_{\varepsilon}\right)}^{2}+\|W\|_{L^{2}\left(\Omega_{\varepsilon}\right)}^{2}\right] \\
& \leq c\left(\frac{-1}{\log (\varepsilon)}\right)^{2}=o\left(\frac{-1}{\log (\varepsilon)}\right) .
\end{aligned}
$$

Then, from the decomposition (24), one can derive

$$
\begin{aligned}
\int_{0}^{T} \int_{\partial \mathcal{O}_{z, \varepsilon}} \sigma\left(v_{\varepsilon}, p_{v_{\varepsilon}}\right) n u_{0} d s d t & =\int_{0}^{T} \int_{\partial \mathcal{O}_{z, \varepsilon}} \sigma\left(z_{\varepsilon}, s_{\varepsilon}\right) n u_{0} d s d t \\
& +\frac{4 \pi v}{\log (\varepsilon)} \int_{0}^{T} w_{0}(z, t)\left(\int_{\partial \mathcal{O}_{z, \varepsilon}} \sigma(E, \Pi)(x-z) n u_{0}(x, t) d s(x)\right) d t,
\end{aligned}
$$

where $\sigma(E, \Pi) n$ is the $2 \times 2$ matrix defined by $(\sigma(E, \Pi) n)_{i, j}=\left(\sigma\left(E^{j}, \Pi^{j}\right) n\right)_{i}, 1 \leq i, j \leq 2$.

Using Theorem 3.3 and the smoothness of $u_{0}$ in $\mathcal{O}_{z, \varepsilon}$, it follows

$$
\left|\int_{0 \partial \mathcal{O}_{z, \varepsilon}}^{T} \sigma\left(z_{\varepsilon}, s_{\varepsilon}\right) n u_{0} d s d t\right|=o\left(\frac{-1}{\log (\varepsilon)}\right) .
$$

The second term in (36) can be written

$$
\begin{aligned}
\int_{\partial \mathcal{O}_{z, \varepsilon}} \sigma(E, \Pi)(x-z) n u_{0}(x, t) d s(x) & =\int_{\partial \mathcal{O}_{z, \varepsilon}} \sigma(E, \Pi)(x-z) n\left[u_{0}(x, t)-u_{0}(z, t)\right] d s(x) \\
& +\int_{\partial \mathcal{O}_{z, \varepsilon}} \sigma(E, \Pi)(x-z) n u_{0}(z, t) d s(x) .
\end{aligned}
$$

Using the trace theorem and the variable change $x=z+\varepsilon y$, one can obtain

$$
\begin{aligned}
& \left|\int_{0}^{T} w_{0}(z, t) \cdot\left(\int_{\partial \mathcal{O}_{z, \varepsilon}} \sigma(E, \Pi)(x-z) n\left[u_{0}(x, t)-u_{0}(z, t)\right] d s(x)\right) d t\right| \\
& \leq c\left\|w_{0}(z, t)\right\|_{L^{2}(0, T)}\|\sigma(E, \Pi)(x-z) n\|_{H^{-1 / 2}\left(\partial \mathcal{O}_{z, \varepsilon}\right)}\left\|u_{0}(x, t)-u_{0}(z, t)\right\|_{L^{2}\left(0, T ; H^{1 / 2}\left(\mathcal{O}_{z, \varepsilon}\right)\right)} .
\end{aligned}
$$

By the fact that $u_{0}$ is smooth in $\mathcal{O}_{z, \varepsilon}$, it follows

$$
\lim _{\varepsilon \longrightarrow 0}\left\|u_{0}(x, t)-u_{0}(z, t)\right\|_{L^{2}\left(0, T ; H^{1 / 2}\left(\mathcal{O}_{z, \varepsilon}\right)\right)}=0 .
$$

Recall that $B(0, r) \subset \mathcal{O}, \mathcal{O}_{r}=\mathcal{O} \backslash \overline{B(0, r)}$ and $\mathcal{O}_{r, \varepsilon}=z+\varepsilon \mathcal{O}_{r} \subset \mathcal{O}_{z, \varepsilon}$. Here, one can check that the function $x \mapsto$ $\sigma(E, \Pi)(x-z)$ is smooth in $\mathcal{O}_{r, \varepsilon}$. Using the trace theorem, we prove that the quantity $\|\sigma(E, \Pi)(x-z) n\|_{H^{-1 / 2}\left(\partial \mathcal{O}_{z, \varepsilon}\right)}$ 
is bounded with respect to $\varepsilon$, which implies

$$
\frac{4 \pi v}{\log (\varepsilon)} \int_{0}^{T} w_{0}(z, t) \cdot\left(\int_{\partial \mathcal{O}_{z, \varepsilon}} \sigma(E, \Pi)(x-z) n\left[u_{0}(x, t)-u_{0}(z, t)\right] d s(x)\right) d t=o\left(\frac{-1}{\log (\varepsilon)}\right) .
$$

Combining the above estimates, one can deduce

$$
\begin{aligned}
& \int_{0}^{T} \int_{\partial \Theta_{z, \varepsilon}} \sigma\left(v_{\varepsilon}, p_{v_{\varepsilon}}\right) n u_{0} d s d t \\
& =\frac{4 \pi v}{\log (\varepsilon)} \int_{0}^{T} w_{0}(z, t) \cdot\left(\int_{\partial \mathcal{O}_{z, \varepsilon}} \sigma(E, \Pi)(x-z) n d s(x)\right) u_{0}(z, t) d t+o\left(\frac{-1}{\log (\varepsilon)}\right) .
\end{aligned}
$$

Since $\operatorname{div}\left(\sigma\left(E^{j}, \Pi^{j}\right)(x-z)\right)=\delta_{z} e_{j}$ in $\mathcal{O}_{z, \varepsilon}$, it follows

$$
\int_{\partial \mathcal{O}_{\varepsilon}} \sigma(E(x-z), \Pi(x-z)) n d s=I,
$$

where $I$ is the $2 \times 2$ identity matrix.

Then, the last estimate becomes

$$
\int_{0}^{T} \int_{\partial \mathcal{O}_{z, \varepsilon}} \sigma\left(v_{\varepsilon}, p_{v_{\varepsilon}}\right) n u_{0} d s d t=\frac{4 \pi v}{\log (\varepsilon)} \int_{0}^{T} w_{0}(z, t) u_{0}(z, t) d t+o\left(\frac{-1}{\log (\varepsilon)}\right) .
$$

Consequently, all shape functions $j$ satisfying the assumption $(\mathcal{A})$ admit the asymptotic expansion

$$
j\left(\Omega \backslash \overline{\mathcal{O}_{z, \varepsilon}}\right)=j(\Omega)+\frac{-1}{\log (\varepsilon)}\left[4 \pi v \int_{0}^{T} w_{0}(z, t) u_{0}(z, t) d t+\delta \mathcal{J}(z)\right]+o\left(\frac{-1}{\log (\varepsilon)}\right) .
$$

\subsubsection{Proof of Proposition 3.8}

Since the desired fluid flow state $\mathcal{W}_{d} \in L^{2}\left(0, T ; H^{1}(\Omega)\right)$, the function $J_{0}$ is differentiable at $w_{0}(., t)$ and we have

$$
D J_{0}\left(w_{0}(., t)\right)(v)=2 \int_{\Omega}\left(w_{0}(., t)-\mathcal{W}_{d}(., t)\right) v d x, \quad \forall v \in H^{1}(\Omega) .
$$

The variation of the associated shape function $j$ is given by

$$
\begin{aligned}
j\left(\Omega_{z, \varepsilon}\right)-j(\Omega) & =\int_{0 \Omega_{z, \varepsilon}} \int_{\Omega_{\varepsilon}}\left|w_{d}\right|^{2} d x d t-\int_{0}^{T} \int_{\Omega}\left|w_{0}-\mathcal{W}_{d}\right|^{2} d x d t \\
& =\int_{0}^{T} D J_{0}\left(w_{0}\right)\left(w_{\varepsilon}-w_{0}\right) d t+\int_{0 \Omega_{z, \varepsilon}}\left|w_{\varepsilon}-w_{0}\right|^{2} d x d t \\
& +\int_{0}^{T} \int_{\mathcal{O}_{z, \varepsilon}}\left|w_{0}\right|^{2} d x d t-\int_{0}^{T} \int_{\mathcal{O}_{z, \varepsilon}}\left|\mathcal{W}_{d}\right|^{2} d x d t .
\end{aligned}
$$

Using the smoothness of $w_{0}$ and $\mathcal{W}_{d}$ in $\Omega$, one can conclude that

$$
\int_{0}^{T} \int_{\mathcal{O}_{z, \varepsilon}}\left|w_{0}\right|^{2} d x d t=o(\varepsilon) \text { and } \int_{0}^{T} \int_{\mathcal{O}_{z, \varepsilon}}\left|\mathcal{W}_{d}\right|^{2} d x d t=o(\varepsilon)
$$


For the two-dimensional case: Using the decomposition (24), it follows

$$
\int_{0}^{T} \int_{\Omega_{z, \varepsilon}}\left|w_{\varepsilon}-w_{0}\right|^{2} \leq 2\left[\int_{0}^{T} \int_{\Omega_{z, \varepsilon}}\left|z_{\varepsilon}\right|^{2} d x d t+\frac{1}{(\log (\varepsilon))^{2}} \int_{0}^{T} \int_{\Omega_{z, \varepsilon}}|W|^{2} d x d t\right] .
$$

From Theorem 3.3, one can check

$$
\int_{0}^{T} \int_{\Omega_{z, \varepsilon}}\left|z_{\varepsilon}\right|^{2} d x d t=o\left(\frac{-1}{\log (\varepsilon)}\right)
$$

Making use of (27), one can deduce

$$
\|W\|_{L^{2}\left(0, T ; L^{2}\left(\Omega_{z, \varepsilon}\right)\right)}=4 \pi v\left\|w_{0}(z, t)\right\|_{L^{2}(0, T)}\|E(x-z)\|_{L^{2}\left(\Omega_{z, \varepsilon}\right)}=O(1) .
$$

Then, it follows

$$
\frac{1}{(\log (\varepsilon))^{2}} \int_{0}^{T} \int_{\Omega_{z, \varepsilon}}|W|^{2} d x d t=o\left(\frac{-1}{\log (\varepsilon)}\right) .
$$

For the three-dimensional case: Using the decomposition (17), it follows

$$
\int_{0}^{T} \int_{\Omega_{z, \varepsilon}}\left|w_{\varepsilon}-w_{0}\right|^{2} \leq 2\left(\int_{0}^{T} \int_{\Omega_{z, \varepsilon}}\left|z_{\varepsilon}\right|^{2} d x d t+\int_{0}^{T} \int_{\Omega_{z, \varepsilon}}|W|^{2} d x d t\right) .
$$

Using Theorem 3.1 and the change of variable, one can check

$$
\int_{0}^{T} \int_{\Omega_{z, \varepsilon}}\left|z_{\varepsilon}\right|^{2} d x d t=o(\varepsilon) \text { and } \int_{0}^{T} \int_{\Omega_{z, \varepsilon}}|W|^{2} d x d t=o(\varepsilon) .
$$

Therefore the function $J_{\varepsilon}$ satisfies the assumption $(\mathcal{A})$ with

$$
\begin{gathered}
D J_{0}\left(w_{0}(., t)\right)(v)=2 \int_{\Omega}\left(w_{0}(., t)-\mathcal{W}_{d}(., t)\right) v d x, \quad \forall v \in H^{1}(\Omega), \\
\delta \mathcal{J}(x)=0, \quad \forall x \in \Omega .
\end{gathered}
$$

\subsubsection{Proof of Proposition 3.9}

The function $J_{0}$ is differentiable at $w_{0}(., t)$ and we have

$$
D J_{0}\left(w_{0}(., t)\right)(v)=2 v \int_{\Omega}\left(\nabla w_{0}(., t)-\nabla \mathcal{W}_{d}(., t)\right) \nabla v d x, \quad \forall v \in H^{1}(\Omega) .
$$

The variation of the associated shape function $j$ is given by

$$
\begin{aligned}
j\left(\Omega_{z, \varepsilon}\right)-j(\Omega) & =\int_{0}^{T} D J_{0}\left(w_{0}\right)\left(w_{\varepsilon}-w_{0}\right) d t-v \int_{0}^{T} \int_{\mathcal{O}_{z, \varepsilon}}\left|\nabla \mathcal{W}_{d}\right|^{2} d x d t \\
& +v \int_{0}^{T} \int_{\mathcal{O}_{z, \varepsilon}}\left|\nabla w_{0}\right|^{2} d x d t+v \int_{0}^{T} \int_{\Omega_{z, \varepsilon}}\left|\nabla w_{\varepsilon}-\nabla w_{0}\right|^{2} d x d t .
\end{aligned}
$$


Thanks to the regularity of $w_{0}$ and $\mathcal{W}_{d}$ in $\mathcal{O}_{z, \varepsilon}$, one can derive

$$
\int_{0}^{T} \int_{\mathcal{O}_{z, \varepsilon}} v\left|\nabla w_{0}\right|^{2} d x d t=o(\varepsilon), \quad \int_{0}^{T} \int_{\mathcal{O}_{z, \varepsilon}} v\left|\nabla \mathcal{W}_{d}\right|^{2} d x d t=o(\varepsilon) .
$$

- For the two-dimensional case: By an adaptation of the technique used in the proof of Theorem 3.7, one can derive

$$
\int_{0 \partial \mathcal{O}_{z, \varepsilon}}^{T} \sigma\left(z_{\varepsilon}, s_{\varepsilon}\right) n w_{0} d s d t=\frac{-4 \pi v}{\log (\varepsilon)} \int_{0}^{T}\left|w_{0}(z, t)\right|^{2} d t+o\left(\frac{-1}{\log (\varepsilon)}\right) .
$$

Therefore, the function $J_{\varepsilon}$ satisfies the assumption $(\mathcal{A})$ with

$$
\begin{gathered}
D J_{0}\left(w_{0}(., t)\right)(v)=2 v \int_{\Omega}\left(\nabla w_{0}(., t)-\nabla \mathcal{W}_{d}(., t)\right) \nabla v d x, \quad \forall v \in H^{1}(\Omega), \\
\text { and } \delta \mathcal{J}(x)=4 \pi v \int_{0}^{T}\left|w_{0}(z, t)\right|^{2} d t, \quad \forall x \in \Omega .
\end{gathered}
$$

- $\quad$ For the three-dimensional case: By an adaptation of the technique used in the proof of Theorem 3.5, one can derive

$$
\int_{0}^{T} \int_{\partial \mathcal{O}_{z, \varepsilon}} \sigma\left(z_{\varepsilon}, s_{\varepsilon}\right) n w_{0} d s d t=\varepsilon\left[\int_{0}^{T} w_{0}(z, t) \cdot \mathcal{M}_{\mathcal{O}} w_{0}(z, t) d t\right]+o(\varepsilon) .
$$

Therefore, the function $J_{\varepsilon}$ satisfies the assumption $(\mathcal{A})$ with

$$
\begin{gathered}
D J_{0}\left(w_{0}(., t)\right)(v)=2 v \int_{\Omega}\left(\nabla w_{0}(., t)-\nabla \mathcal{W}_{d}(., t)\right) \nabla v d x, \quad \forall v \in H^{1}(\Omega), \\
\text { and } \delta \mathcal{J}(z)=\int_{0}^{T} w_{0}(z, t) . \mathcal{M} \mathcal{M}_{\mathcal{O}} w_{0}(z, t) d t, \forall z \in \Omega .
\end{gathered}
$$

\section{Conclusion}

This paper deals with non-stationary Navier-Stokes topological optimization problem. In the theoretical part of this work, we have established a topological asymptotic formula describing the shape function variation related to a small Dirichlet geometric perturbation.

The obtained theoretical results are exploited for building a topological optimization algorithm for solving the Tesla micro-valve optimization problem. We illustrate the strengths of this approach namely the ability to find optimal design based only on boundary conditions and constraints information without the need of an initial design.

Acknowledgement: The authors acknowledge funding from the Research and Development (R\&D) Program (Research Pooling Initiative), Ministry of Education, Riyadh, Saudi Arabia, (RPI-KSU). We are very grateful to Professor Maatoug Hassine for the interesting discussions that have improved the quality of this document. 


\section{References}

[1] N. Tesla, Valvular Conduit, U.S. Patent NO. 1.329.559, 1920.

[2] C.J. FG Morris, Electronic cooling systems based on fixed-valve micropump networks, Transducers Research Foundation, Cleveland Heights, Ohio, 2000.

[3] M. Wackerle, H.J. Bigus and T.V. Blumenthal, Micro pumps for lab technology and medicine, Final presentation of the project ì-DOS, Fraunhofer IZM, Munich, 2006.

[4] S. Shoji and M. Esashi, Microflow devices and systems, Journal of Micromechanics and Microengineering 4 (1994), $157-$ 171.

[5] G.T.A. Kovacs, Micromachined transducers sourcebook, New York, Mc Graw-Hill, 839-855, 1998.

[6] E. Morganti and G.U. Pignatel, Microfluidics for the treatment of the hydrocephalus, 1st International Conference on Sensing Technology, Palmerston North, New Zealand (2005), 483-487.

[7] H.J Yoon, J.M. Jung, J.S. Jeong and S.S. Yang, Micro devices for a cerebrospinal fluid (CSF) shunt system, Sensors and Actuators 110, (2004), 68-76.

[8] F. K. Forster, R. L. Bardell, M. A. Afromowitz, N.R. Sharma and A. Blanchard, Design, Fabrication and Testing of Fixed-Valve Micro-Pumps, The ASME Fluid Engineering Division 234, (1995), 39-44.

[9] T.Q. Truong and N.T. Nguyen, Simulation and Optimization of Tesla Valves, 2003 Nanotech - Nanotechnology Conference and Trade Show, San Francisco, CA (2003), 178-181.

[10] R. L. Bardell, The Diodicity Mechanism of Tesla-Type No-Moving-Parts Valves, Ph.D. thesis, University of Washington, Seattle, 2000.

[11] A. R. Gamboa, C. J. Morris and F. K. Forster, Improvements in Fixed-Valve Micropump Performance Through Shape Optimization of Valves, ASME J. Fluids Eng. 127 (2005), no. 2, 339-347.

[12] G. Pingen, A. Evgrafov and K. Maute, A Parallel Schur Complement Solver for the Solution of the Adjoint Steady-State Lattice Boltzmann Equations: Application to Design Optimisation, Int. J. Comput. Fluid Dyn. 22 (2008), no. 7, $457-464$.

[13] S. Lin, Y. Deng, Y. Wu and Z. Liu, Optimizing Tesla valve for inertial microfluidics, The third international conference of advances in microfluidics and nanofluidics, Dalian, China, 2012.

[14] T. Borrvall and J. Petersson, Topology optimization of fuids in stokes flow, Int. J. Numer. Meth. Fluids 41 (2003), 77-107.

[15] S. Lin, L. Zhao, J. K. Guest, T. P. Weihs and Z. Liu, Topology Optimization of Fixed-Geometry Fluid Diodes, ASME Journal of Mechanical Design 137 (2015), no 8, 081402.

[16] M. Abdelwahed and M. Hassine, Topological optimization method for a geometric control problem in Stokes flow, Appl. Numer. Math. 59 (2009), 1823-1838.

[17] S. Amstutz, The topological asymptotic for the Navier-Stokes equations, ESAIM, Control, Optim. and Cal. of Variat. 11 (2005), 401-425.

[18] M. Hassine, Shape optimization for the Stokes equations using topological sensitivity analysis, ARIMA 5 (2006), 216-229.

[19] M. Hassine and M. Masmoudi, The topological asymptotic expansion for the quasi-Stokes problem, ESAIM. Control, Optimisation and Calculus of Variations 10 (2004), no. 4, 478-504.

[20] R. L. Panton, Incompressible Flow, John Wiley and Sons, New York, NY, USA, 1984.

[21] R. Temam, Navier-Stokes equations. AMS Chelsea Publishing, Providence, RI, Theory and numerical analysis, Reprint of the 1984 edition, 2001.

[22] R. Dautray and J.L. Lions, Analyse mathématique et calcul numérique pour les sciences et les techniques, Masson, collection CEA 6,1987.

[23] X. Duan, Y. Ma, R. Zhang, Shape-topology optimization for Navier-Stokes problem using variational level set method, J. Comput. Appl. Math. 222 (2008), 487-499.

[24] M. Abdelwahed and M. Hassine, Topology Optimization of Time Dependent Viscous Incompressible Flows, Abstract and Applied Analysis (2014), Article ID 923016.

[25] O. Pironneau, On optimum profiles in Stokes flow, J. Fluid Mech. 59 (1973), no. 1, 117-128.

[26] S.M. Thompson, B. J. Paudel, T. Jamal and D. K. Walters, Numerical Investigation of Multistaged Tesla Valves, Journal of Fluids Engineering 136 (2014), no. 8, 081102.

[27] P. Guillaume and K. Sidi Idris, Topological sensitivity and shape optimization for the Stokes equations, SIAM Journal on Control and Optimization 43 (2004), no. 1, 1-31.

[28] G. Allaire and M. Schoenauer, Conception optimale de structures, Mathématiques et Applications, volume 58, Springer, 2007. 